\title{
SEPARATION AND CODING
}

\author{
STEPHEN WATSON
}

\begin{abstract}
We construct a normal collectionwise Hausdorff space which is not collectionwise normal with respect to copies of $[0,1]$. We do this by developing a general theory of coding properties into topological spaces. We construct a para-Lindelöf regular space in which para-Lindelöf is coded directly rather than $\sigma$-para-Lindelöf and normal. We construct a normal collectionwise Hausdorff space which is not collectionwise normal in which collectionwise Hausdorff is coded directly rather than obtained as a side-effect to countable approximation. We also show that the Martin's axiom example of a normal space which is not collectionwise Hausdorff is really just a kind of "dual" of Bing's space.
\end{abstract}

\section{INTRODUCTION}

In this paper, we construct examples in which properties such as collectionwise Hausdorff or para-Lindelöf are built directly into the example. In some cases, examples with the stated properties have already been described in the literature. Navy's space [20] is para-Lindelöf because normality and $\sigma$-paraLindelöf have been built in. Here, para-Lindelöf itself is built in. Fleissner's example George [11] is collectionwise Hausdorff because of a construction by countable approximation. Here Bing's construction [2] is applied to copies of $\omega_{1}$, for example, and collectionwise Hausdorff is built in directly. In particular, a question of Fleissner and Reed is answered by constructing a normal collectionwise Hausdorff space which is not collectionwise normal with respect to copies of the closed unit interval. We also show that the Martin's axiom example of a normal space which is not collectionwise Hausdorff is really just a kind of "dual" of Bing's space. The general technique is presented because building a property directly into a space is a method which is more likely to preserve the failure of other properties. We argue that the methods in use fall into two groups corresponding to the logical connectives AND and OR. We also present some methods which take a space and produce another space which has similar separation properties but which has additional properties such as metacompact, locally collectionwise normal and perfect.

Received by the editors December 3, 1986.

1991 Mathematics Subject Classification. Primary 54D15, 54G20; Secondary 54D18, 54G15, 54E30.

This work has been supported by the Natural Sciences and Engineering Research Council of Canada. 


\section{AND AND OR CONSTRUCTIONS}

These constructions can be classified into two types: AND and OR constructions. When a neighborhood is assigned to a nonisolated point, it is useful to imagine that the point is specifying certain other points whose neighborhoods its neighborhood does not wish to intersect. In an AND construction, two neighborhoods will actually not intersect if each point specifies the other. In an OR construction, two neighborhoods will actually not intersect if either point specifies the other. When more than one property is built into a space, the construction can be a combination of two AND constructions or two OR constructions or be a hybrid AND-OR construction. The choice of construction depends on the circumstances. An AND construction is less likely to cause too much separation but an OR construction is more likely to allow a lowering of character or create a clopen base for the topology.

Some of the examples to be defined are similar to well-known examples not in the sense that they are homeomorphic but in a weaker sense. A definition is needed to make this precise.

Definition 1. Two topological spaces $X$ and $Y$ are said to have identical separation properties if $X^{\prime}=Y^{\prime}$ and if, for each discrete family $\left\{A_{\alpha}: \alpha \in \kappa\right\}$ in $X^{\prime},\left\{A_{\alpha}: \alpha \in \kappa\right\}$ is separated in $X$ if and only if $\left\{A_{\alpha}: \alpha \in \kappa\right\}$ is separated in $Y$.

Let us illustrate these ideas with the simplest example of an AND construction, Bing's space.

Example 1. Let $J=\left\{\{(\alpha, P),(\beta, Q)\} \in\left[\omega_{1} \times\left[\mathscr{P}\left(\omega_{1}\right)\right]^{<\omega}\right]^{2}:(\forall A)(A \in P \cap\right.$ $Q) \Rightarrow(\alpha \in A \Leftrightarrow \beta \in A)\}$. Let $X=\omega_{1} \cup J$ be topologized by letting $J$ be a set of isolated points and letting a neighborhood of $\alpha \in \omega_{1}$ be defined in each parameter $E \in\left[\mathscr{P}\left(\omega_{1}\right)\right]^{<\omega}$ by letting $U_{E}(\alpha)=\{\alpha\} \cup\{\{(\alpha, P),(\beta, Q)\} \in J$ : $P \supset E\}$.

We are using the Erdös notations $[\kappa]^{\lambda}=\{A \subset \kappa:|A|=\lambda\}$. The notations $[\kappa]^{<\lambda}$ and $[\kappa] \leq \lambda$ are self-explanatory. We describe the reason that Example 1 is an AND construction. Neighborhoods of the point $\alpha$ are defined in a parameter which is a finite subset of $\mathscr{P}\left(\omega_{1}\right)$. Any isolated point which lies in such a neighborhood must have a corresponding second coordinate which contains this finite subset. If neighborhoods of $\alpha_{0}$ and $\alpha_{1}$ intersect, then they contain a common isolated point. If the neighborhoods use a common element $A$ of $\mathscr{P}\left(\omega_{1}\right)$, then the second coordinates must both contain $A$. Thus $A$ must satisfy the condition $\alpha \in A \Leftrightarrow \beta \in A$. This condition applies to every isolated point in the intersection of the neighborhoods if and only if both neighborhoods are defined in a parameter which contains $A$.

Lemma 1. $X$ is a normal space which is not collectionwise Hausdorff.

Proof. This is not the traditional presentation but this example and Bing's example $G$ (when $P=\omega_{1}$ ) in [2] have identical separation properties. The difference in presentation lies in the dependence here of the intersection of neighborhoods on the existence of isolated points. The proof is just the proof of Lemma 2 in the special case where $Z$ is $\omega_{1}$ with the discrete topology and $\mathrm{BO}(Z)=\left\{\{\alpha\}: \alpha \in \omega_{1}\right\}$. 
In [31], we showed that Example 1 remains normal when $\mathscr{P}\left(\omega_{1}\right)$ is replaced by a subfamily $\mathscr{A}$ precisely when $\mathscr{A}$ generates $\mathscr{P}\left(\omega_{1}\right)$ under finite Boolean operations and then countable unions. This is a very difficult condition for $\mathscr{A}$ to satisfy without being the same cardinality as $\mathscr{P}\left(\omega_{1}\right)$ (although not impossible, see [31]). In fact, we left two questions open:

Problem 1. Does $2^{\aleph_{0}}<2^{\aleph_{1}}$ imply that there is no family of size less than $2^{\aleph_{1}}$ which generates the power set of $\omega_{1}$ under countable unions?

Problem 2. Is $2^{\aleph_{0}}=2^{\aleph_{1}}=\aleph_{3}$ consistent with the existence of a family of size $\aleph_{2}$ which generates the power set of $\omega_{1}$ under countable unions?

These questions remain of interest. In fact, Steprāns [25] and independently Jech and Prikry [17] showed that the answer to Problem 1 is yes so long as either $2^{\aleph_{0}}<\aleph_{\omega_{1}}$ holds or else $2^{\aleph_{0}}=\kappa,\left(\kappa^{+}\right)^{L}<2^{\aleph_{1}}$ and the covering lemma over $L$ is true. These partial results would make a solution to Problem 1 in either direction quite impressive.

The reader might think that a model of $\mathrm{MA}_{\aleph_{1}}$ would be the right place to look for a solution of Problem 2. However $M_{\aleph_{\aleph_{1}}}$ implies that the minimal size of such a family of $2^{\aleph_{1}}$. The model where $\aleph_{\omega}$ Cohen reals are added to a model of GCH has such a subfamily of cardinality $\aleph_{\omega}<2^{\aleph_{0}}$ but this is an unsatisfying result.

A general AND construction which codes normality is useful to define as it will be incorporated into other examples later. We will use $\operatorname{CO}(X)$ to indicate the family of clopen subsets of a topological space $X$ and we will use $\operatorname{BO}(X)$ to indicate an arbitrary base of clopen sets for $X$.

Example 2. Let $Z$ be a normal strongly 0-dimensional space (strong 0-dimensionality is a hypothesis which only simplifies the exposition). Let

$$
\begin{aligned}
& J=\{\{(U, P),(V, Q)\} \in\left[\mathrm{CO}(Z) \times[\mathrm{CO}(Z)]^{<\omega}\right]^{2}: \\
&(\forall A)(A \in P \cap Q) \Rightarrow(U \subset A \Leftrightarrow V \subset A)\} .
\end{aligned}
$$

Let $X(Z)=Z \cup J$ be topologized by letting $J$ be a set of isolated points and letting an open set $U_{E}$ be defined in each parameter $U \in \mathrm{BO}(Z)$ and $E \in[\mathrm{CO}(Z)]^{<\omega}$ by letting

$$
U_{E}=U \cup\{\{(V, P),(W, Q)\} \in J: P \supset E, V \subset U\} .
$$

Lemma 2. $X(Z)$ is normal and is collectionwise Hausdorff if and only if $Z$ is $\aleph_{1}$-compact.

Proof. Since $U \subset U^{\prime}, E \supset E^{\prime} \Rightarrow U_{E} \subset U_{E^{\prime}}^{\prime}$ and $U_{E} \cap Z=U$ and $\cap\left\{U_{E}\right.$ : $\left.E \in[\mathrm{CO}(Z)]^{<\omega}\right\}=U$, we know that $X(Z)$ is a $T_{1}$ topological space which induces a subspace topology on $Z$ which is identical to the original topology. To see that $X(Z)$ is normal, it suffices to show that a clopen set $A \subset Z$ can be separated from $Z-A$. We claim that

$$
\bigcup\left\{U_{\{A\}}: U \in \mathrm{BO}(Z), U \subset A\right\} \cap \bigcup\left\{U_{\{A\}}: U \in \mathrm{BO}(Z), U \cap A=\varnothing\right\}=\varnothing .
$$

If $\{(V, P),(W, Q)\} \in\left(U_{0}\right)_{\{A\}} \cap\left(U_{1}\right)_{\{A\}}$ where $U_{0}, U_{1} \in \mathrm{BO}(Z), U_{0} \subset A$, and $U_{1} \cap A=\varnothing$, then suppose, without loss of generality, that $V \subset U_{0}$ and $W \subset U_{1}$. Since $A \in P \cap Q$, we get $V \subset A \Leftrightarrow W \subset A$ which is a contradiction. If $Z$ contains an uncountable closed discrete set, then it must contain an 
uncountable disjoint family $\left\{U_{E^{\alpha}}^{\alpha}: \alpha \in \omega_{1}\right\}$ of open sets. We assume, without loss of generality, that $\left\{E^{\alpha}: \alpha \in \omega_{1}\right\}$ is a $\Delta$-system with root $\Delta$. We can find $\alpha_{0}, \alpha_{1}$ such that $F \in \Delta$ implies that $U^{\alpha_{0}} \subset F \Leftrightarrow U^{\alpha_{1}} \subset F$. Now $\left\{\left(U^{\alpha_{0}}, E_{\alpha_{0}}\right),\left(U^{\alpha_{1}}, E_{\alpha_{1}}\right)\right\} \in U_{E^{\alpha_{0}}}^{\alpha_{0}} \cap U_{E^{\alpha_{1}}}^{\alpha_{1}}$. Of course, if $Z$ is $\aleph_{1}$-compact, then regular spaces are $\aleph_{0}$-collectionwise Hausdorff.

There is an OR analogue of Example 1.

Example 3. Let $J=\left\{\{(\alpha, P),(\beta, Q)\} \in\left[\omega_{1} \times\left[\mathscr{P}\left(\omega_{1}\right)\right]^{<\omega}\right]^{2}:(\forall A)(A \in P \cup\right.$ $Q) \Rightarrow(\alpha \in A \Leftrightarrow \beta \in A)\}$. Let $Y=\omega_{1} \cup J$ be defined and topologized as in Example 1 .

We describe the reason that Example 3 is an OR construction. Neighborhoods of the point $\alpha$ are defined in a parameter which is a finite subset of $\mathscr{P}\left(\omega_{1}\right)$. Any isolated point which lies in such a neighborhood must have a corresponding second coordinate which contains this finite subset. If neighborhoods of $\alpha_{0}$ and $\alpha_{1}$ intersect, then they contain a common isolated point. If either of the neighborhoods use a particular element $A$ of $\mathscr{P}\left(\omega_{1}\right)$, then at least one of the second coordinates must contain $A$. Thus $A$ must satisfy the condition $\alpha \in$ $A \Leftrightarrow \beta \in A$. This condition applies to every isolated point in the intersection of the neighborhoods if and only if at least one neighborhood is defined in a parameter which contains $A$.

Unfortunately, $Y$ is paracompact.

Lemma 3. $Y$ is a paracompact regular space which is the free union of spaces with one nonisolated point.

Proof. This space $Y$ is actually a closed subspace of $X$. The only difference is that the restriction on what it means to be an isolated point is stronger for $Y$. Thus $Y$ is a Hausdorff space. Let us calculate

$$
\begin{aligned}
U_{\{\alpha\}}(\alpha)= & \{\alpha\} \cup\{\{(\alpha, P),(\beta, Q)\}:(A \in P \cup Q \Rightarrow(\alpha \in A \Leftrightarrow \beta \in A)), \\
& (P \supset\{\alpha\}) \vee Q \supset\{\alpha\})\} \\
= & \{\alpha\} \cup\{\{(\alpha, P),(\alpha, Q)\}: \alpha \in P \cup Q\} .
\end{aligned}
$$

This means that $\alpha_{0} \neq \alpha_{1} \Rightarrow U_{\left\{\alpha_{0}\right\}}\left(\alpha_{0}\right) \cap U_{\left\{\alpha_{1}\right\}}\left(\alpha_{1}\right)=\varnothing$.

We can, however, make a modification analogous to that which was made in [31]. We ask whether $\mathscr{P}\left(\omega_{1}\right)$ in Example 3 can be replaced by some subfamily $\mathscr{A}$ of smaller cardinality so that the resulting space $Y(\mathscr{A})$ remains normal but is no longer collectionwise Hausdorff.

Lemma 4. Let $\mathscr{A}$ be a countable subset of $P\left(\omega_{1}\right)$. The following are equivalent:

1. $Y(\mathscr{A})$ is completely regular but not collectionwise Hausdorff.

2. $Y(\mathscr{A})$ is Hausdorff.

3. $\mathscr{A}$ is a point-separating family.

4. $\mathscr{A}$ is a clopen subbase for a zero-dimensional separable metrizable topology $\tau$ on $\omega_{1}$.

Proof. We show only that $(4) \Rightarrow(1)$. Let $E \in[\mathscr{A}]^{<\omega}$ and $\alpha \in \omega_{1}$. The open set $U_{E}(\alpha)$ is closed since $|A \cap\{\alpha, \beta\}|=1 \Rightarrow U_{\{A\}}(\beta) \cap U_{E}(\alpha)=\varnothing$. Suppose $E_{\alpha} \in[\mathscr{A}]^{<\omega}$ is defined for each $\alpha \in \omega_{1}$. Let $I_{\alpha}: E_{\alpha} \rightarrow 2$ be defined by $I_{\alpha}(T)=1 \Leftrightarrow \alpha \in T$. We use the convention that $A^{1}=A$ and $A^{0}=A^{c}$. Let 
$F_{\alpha}=\bigcap\left\{T^{I_{\alpha}(T)}: T \in E_{\alpha}\right\}$. Each $F_{\alpha}$ is a clopen neighborhood of $\alpha$. In any separable metrizable topology, we can find $\alpha, \beta$ such that $\{\alpha, \beta\} \subset F_{\alpha} \cap F_{\beta}$.

Thus the standard normal first countable not collectionwise Hausdorff space [29] (the bubble space over a $Q$-set) is the OR variant of Bing's space with the character lowered in the simplest possible way:

Lemma $5\left(\mathrm{MA}_{\aleph_{1}}\right)$. Let $\mathscr{A}$ be a countable subset of $\mathscr{P}\left(\omega_{1}\right)$. If $Y(\mathscr{A})$ is Hausdorff then $Y(\mathscr{A})$ is normal.

Proof. Let $A$ be a $\tau$-basic open set. We can express $A=\bigcap\left\{A_{i}^{I(i)}: i \in n\right\}$ where $n \in \omega$ and $I: n \rightarrow 2$. Let $f_{A}: \omega_{1} \rightarrow[\mathscr{A}]^{<\omega}$ be defined by $f_{A} \uparrow A \equiv\left\{A_{i}: i \in n\right\}$ and $f_{A}\left\lceil\left(\omega_{1}-A\right) \equiv \varnothing\right.$. We have $f_{A}$ separating $A$ from $\omega_{1}-A$. Let $B$ be a $\tau$-open set. We can express $B=\bigcup\left\{B_{i}: i \in \omega\right\}$ where each $B_{i}$ is $\tau$-basic open. For each $\alpha \in B$, let $n(\alpha)=\min \left\{n \in \omega: \alpha \in B_{i}\right\}$. Let $f_{B}: \omega_{1} \rightarrow[\mathscr{A}]^{<\omega}$ be defined by $f_{B}(\alpha)=f_{B_{n(\alpha)}}(\alpha)$ where $\alpha \in B$ and $f_{B}(\alpha)=\varnothing$ otherwise. Now $f_{B}$ separates $B$ from $\omega_{1}-B$, i.e., any closed set from its complement.

We need a classical lemma from the 1920s (see also Lemma 1.5.14 of [7]):

Lemma 6 (Urysohn [30]). If $X$ is a $T_{1}$ space and for every pair of disjoint closed sets $F_{0}, F_{1}$, there is a countable family of open subsets of $X$ which cover $F_{0}$ and none of the closures of which intersect $F_{1}$, then the space $X$ is normal.

Proof of Lemma 5 continued. Let $C$ be a $F_{\sigma}$-set in the sense of $\tau$. The fact that we can separate any $\tau$-closed subset of $\omega_{1}$ from its complement, together with Lemma 6 , implies that we can separate any $\tau-F_{\sigma}$-subset of $\omega_{1}$ from its complement. Under $\mathrm{MA}_{\aleph_{1}}$, any subset of $\omega_{1}$ is an $F_{\sigma}$ in any separable metrizable topology and so $Y(\mathscr{A})$ is normal. The proof of Lemma 5 is complete.

Let us continue with a construction in which normal and $\aleph_{1}$-collectionwise Hausdorff are built in. This construction has two variants: AND/AND and AND/OR. Normality is built in with an AND construction in each variant but $\aleph_{1}$-collectionwise Hausdorff can be built in with either an AND construction or an OR construction. We present the construction of both variations. That is, we present $X_{1}$, the AND/AND variation and $X_{2}$, the AND/OR variation. We also present $X_{0}$, which has identical separation properties to Bing's space on $\kappa$. The AND/OR variation does possess an interesting covering property which the AND/AND variation does not possess. A definition is necessary to express this property.

Definition 2. Let $X$ be a topological space. If every open cover of $X$ has an open refinement, any $\kappa$ many elements of which are a locally finite family, then we say that $X$ is a covering $P_{\kappa}$-space. We call a covering $P_{\omega}$-space simply a covering $P$-space.

Lemma 7. Let $\kappa$ be an infinite cardinal. Any paracompact space is a covering $P_{\kappa}$ space and any covering $P_{\kappa}$-space is $\kappa$-collectionwise Hausdorff and metacompact.

Proof. Suppose that $D=\left\{x_{\alpha}: \alpha \in \kappa\right\}$ is a closed discrete subset of a covering $P_{\kappa}$-space $X$. Let $\left\{U_{\alpha}: \alpha \in \kappa\right\}$ be a family of open sets such that $U_{\alpha} \cap D=\left\{x_{\alpha}\right\}$. Let $\mathscr{U}$ be a cover of $X$ which is the union of $\left\{U_{\alpha}: \alpha \in \kappa\right\}$ and $\{X-D\}$. Let $\mathscr{V}$ be an open refinement of $\mathscr{U}$ any $\kappa$-sized subfamily of which is a locally finite family. For each $\alpha \in \kappa$, choose $V_{\alpha} \in \mathscr{V}$ which contains $x_{\alpha}$. These $V_{\alpha}$ 's are all distinct and $\alpha_{0} \neq \alpha_{1} \Rightarrow \alpha_{0} \notin V_{\alpha_{1}}$. Furthermore, $\left\{V_{\alpha}: \alpha \in \kappa\right\}$ is a locally 
finite family. For each $\alpha \in \kappa$, choose an open neighborhood $W_{\alpha} \subset V_{\alpha}$ of $x_{\alpha}$ such that $\left|\left\{\alpha^{\prime} \in \kappa: W_{\alpha} \cap V_{\alpha^{\prime}} \neq \varnothing\right\}\right|<\omega$. We can partition $\kappa$ into countable equivalence classes and apply regularity to obtain a separation of $\left\{x_{\alpha}: \alpha \subset \kappa\right\}$.

Example 4. Let $\kappa$ be a cardinal. Let $J_{i}$ be the set of $\{(\alpha, P, R),(\beta, Q, S)\} \in$ $\left[\kappa \times[\mathscr{P}(\kappa)]^{<\omega} \times\left[[\kappa]^{2}\right]^{\leq \omega_{1}}\right]^{2}$ which satisfy:

$$
\begin{aligned}
& (A \in P \cap Q) \Rightarrow(\alpha \in A \Leftrightarrow \beta \in A) \\
& \neg(\{\alpha, \beta\} \in R \cap S) \text { for } i=1 \text { only } \\
& \neg(\{\alpha, \beta\} \in R \cup S) \text { for } i=2 \text { only }
\end{aligned}
$$

Let $X_{i}=\kappa \cup J_{i}$. A topology is defined on $X_{i}$ by letting $J_{i}$ be a set of isolated points and letting a neighborhood of $\alpha \in \kappa$ be defined in each $M \in[\mathscr{P}(\kappa)]^{<\omega}$ and $N \in\left[[\kappa]^{2}\right]^{\leq \omega_{1}}$ by

$$
U_{M, N}(\alpha)=\{\alpha\} \cup\left\{\{(\alpha, P, R),(\beta, Q, S)\} \in J_{i}: P \supset M, R \supset N\right\} .
$$

Lemma 8. The topological space $X_{1}$ is a normal $\aleph_{1}$-collectionwise Hausdorff space which is paracompact when $\kappa \leq \omega_{1}$ but otherwise is not $\aleph_{1}$-collectionwise normal with respect to discrete sets of cardinality $\aleph_{2}$. Furthermore, $X_{1}$ is not even a covering $P$-space. The topological space $X_{2}$ is a normal $\aleph_{1}$-collectionwise Hausdorff space which is paracompact when $\kappa \leq \omega_{2}$ but otherwise is not $\aleph_{1}$ collectionwise normal with respect to discrete sets of cardinality $\aleph_{3}$. Furthermore $X_{2}$ is a covering $P_{\aleph_{1}}$-space.

Proof. The proof that $X_{0}$ is a $T_{1}$ topological space which induces the discrete topology on $\kappa$ is similar to that in the proof of Lemma 2. We note that $X_{2}$ is a closed subspace of $X_{1}$ and that, in turn, $X_{1}$ is a closed subspace of $X_{0}$. Furthermore, we note that $X_{0}$ has identical separation properties to the $X$ of Example 1 done instead on $\kappa$ instead of $\omega_{1}$. The only difference is that each isolated point has been replaced by a copy of $\left[\left[[\kappa]^{2}\right]^{\leq \omega_{1}}\right]^{2}$ with the discrete topology. To prove this, note that if $\left\{\left(\beta_{0}, P\right),\left(\beta_{1}, Q\right)\right\} \in U_{M_{0}}\left(\beta_{0}\right) \cap U_{M_{1}}\left(\beta_{1}\right)$ in $X$, then $\left\{\left(\beta_{0}, P, N_{0}\right),\left(\beta_{1}, Q, N_{1}\right)\right\} \in U_{M_{0}, N_{0}}\left(\beta_{0}\right) \cap U_{M_{1}, N_{1}}\left(\beta_{1}\right)$ in $X_{0}$. Also note that if $\left\{\left(\beta_{0}, P, R\right),\left(\beta_{1}, Q, S\right)\right\} \in U_{M_{0}, \varnothing}\left(\beta_{0}\right) \cap U_{M_{1}, \varnothing}\left(\beta_{1}\right)$ in $X_{0}$, then $\left\{\left(\beta_{0}, P\right),\left(\beta_{1}, Q\right)\right\} \in U_{M_{0}}\left(\beta_{0}\right) \cap U_{M_{1}}\left(\beta_{1}\right)$ in $X$. All of this means that, since $X$ is normal, $X_{0}$ is also normal. This implies that, as closed subspaces, $X_{1}$ and $X_{2}$ are also normal.

Next, we show that $X_{1}$ is $\aleph_{1}$-collectionwise Hausdorff. We show simultaneously that $X_{2}$ has the stronger property of being a covering $P_{\aleph_{1}}$-space. We do the latter by showing that any $\aleph_{1}$ many elements of $\left\{U_{\varnothing, \varnothing}(\alpha): \alpha \in \kappa\right\}$ are a locally finite family. Let $D=\left\{\alpha_{\gamma}: \gamma \in \omega_{1}\right\}$ be a subset of $\kappa$. Let $N=\left\{\left\{\alpha_{\gamma}, \alpha_{\gamma^{\prime}}\right\}: \gamma, \gamma^{\prime} \in \omega_{1}, \gamma \neq \gamma^{\prime}\right\}$. In the case of $X_{1}$, we claim that $\left\{U_{\varnothing, N}\left(\alpha_{\gamma}\right): \gamma \in \omega_{1}\right\}$ is a disjoint family of open sets which separate $D$. In the case of $X_{2}$, we claim that $\left\{U_{\varnothing, N}\left(\alpha_{\gamma}\right): \gamma \in \omega_{1}\right\}$ witnesses the fact that the family is discrete. Suppose otherwise that $\left\{\left(\alpha_{\gamma}, P, R\right),\left(\alpha_{\gamma^{\prime}}, Q, S\right)\right\}$ is an element of $U_{\varnothing, N}\left(\alpha_{\gamma}\right) \cap U_{\varnothing, N}\left(\alpha_{\gamma^{\prime}}\right)$ in the case of $X_{1}$ and an element of $U_{\varnothing, N}\left(\alpha_{\gamma}\right) \cap U_{\varnothing, \varnothing}\left(\alpha_{\gamma^{\prime}}\right)$ in the case of $X_{2}$. Since $\left\{\alpha_{\gamma}, \alpha_{\gamma^{\prime}}\right\} \in N$ and $N \subset R \cap S$ in the case of $X_{1}$ while $N \subset R \subset R \cup S$ in the case of $X_{2}$, we get that the isolated point is not an element of $J_{1}$ or $J_{2}$ respectively.

Next, we show that $X_{1}$ is paracompact when $\kappa \leq \omega_{1}$ and that $X_{2}$ is paracompact when $\kappa \leq \omega_{2}$. If $\kappa \leq \omega_{1}$, let $N=\left\{\left\{\alpha, \alpha^{\prime}\right\}: \alpha \in \kappa\right\}$. If $\kappa \leq \omega_{2}$, let $N_{\alpha}=\left\{\left\{\alpha, \alpha^{\prime}\right\}: \alpha^{\prime}<\alpha\right\}$. Observe that $U_{\varnothing, N}(\alpha) \cap U_{\varnothing, N}\left(\alpha^{\prime}\right)=\varnothing$ in $X_{1}$ while $U_{\varnothing, N_{\alpha}}(\alpha) \cap U_{\varnothing, N_{\alpha^{\prime}}}\left(\alpha^{\prime}\right)=\varnothing$ in $X_{2}$. 
Next, we show the failure of specific kinds of collectionwise normality simultaneously for $X_{1}$ and $X_{2}$. Let $\left\{C_{\alpha}: \alpha \in \omega_{1}\right\}$ be disjoint elements of $[\kappa]^{\kappa}$. We show that this family is not separated if $\kappa$ is large enough. Suppose, otherwise, that there are $\left\{A_{\gamma}, B_{\gamma}: \gamma \in \bigcup\left\{C_{\alpha}: \alpha \in \omega_{1}\right\}\right\}$ such that, whenever $\gamma \in C_{\alpha}$, $\gamma^{\prime} \in C_{\alpha^{\prime}}$ and $\alpha \neq \alpha^{\prime}$, we have $U_{A_{\gamma}, B_{\gamma}}(\gamma) \cap U_{A_{\gamma^{\prime}}, B_{\gamma^{\prime}}}\left(\gamma^{\prime}\right) \neq \varnothing$. We need some assumptions about $\kappa$ for the " $\Delta$-system and thinning out" argument which follows. We assume that $\kappa$ is a regular cardinal greater than $\omega_{1}$ (actually we need $\kappa>\omega$ for (6), $\kappa>\omega_{1}$ for (5), and $\operatorname{cf}(k)>\omega$ for (1)). Assume, without loss of generality, that:

1. $\left\{A_{\gamma}: \gamma \in C_{\alpha}\right\}$ is a $\Delta$-system with root $r_{\alpha}$ for each $\alpha \in \omega_{1}$.

2. $\gamma, \gamma^{\prime} \in C_{\alpha} \wedge a \in r_{\alpha} \Rightarrow\left(\gamma \in a \Leftrightarrow \gamma^{\prime} \in a\right)$.

3. $\left\{r_{\alpha}: \alpha \in \omega_{1}\right\}$ is a $\Delta$-system with root $r$.

4. $\gamma, \gamma^{\prime} \in \bigcup\left\{C_{\alpha}: \alpha \in \omega_{1}\right\}$ and $a \in r \Rightarrow\left(\gamma \in a \Leftrightarrow \gamma^{\prime} \in a\right)$.

5. $\alpha \in \omega_{1} \wedge \gamma \in C_{\alpha} \Rightarrow \bigcup\left\{r_{\beta}: \beta \in \omega_{1}\right\} \cap\left(A_{\gamma}-r_{\alpha}\right)=\varnothing$.

6. $\gamma \in C_{\alpha} \wedge \gamma^{\prime} \in C_{\alpha^{\prime}} \wedge \alpha \neq \alpha^{\prime} \Rightarrow A_{\gamma} \cap A_{\gamma^{\prime}}=r$.

This argument has removed the first parameter of the neighborhoods from our consideration. We have "decoded" normality.

Indeed, we argue that, whenever $\gamma \in C_{\alpha}, \gamma^{\prime} \in C_{\alpha^{\prime}}$, and $\alpha \neq \alpha^{\prime}$, we have $U_{\varnothing, B_{\gamma}}(\gamma) \cap U_{\varnothing, B_{\gamma^{\prime}}}\left(\gamma^{\prime}\right)=\varnothing$. Suppose not, that $\left\{(\gamma, P, R),\left(\gamma^{\prime}, Q, S\right)\right\}$ is in that intersection. This implies that $\left\{(\gamma, \varnothing, R),\left(\gamma^{\prime}, \varnothing, S\right)\right\}$ also lies in the intersection. We claim that $\left\{\left(\gamma, A_{\gamma}, R\right),\left(\gamma^{\prime}, A_{\gamma^{\prime}}, S\right)\right\} \in U_{A_{\gamma}, B_{\gamma}}(\gamma) \cap U_{A_{\gamma^{\prime}}, B_{\gamma^{\prime}}}\left(\gamma^{\prime}\right)$. The only reason that this could fail is if there was $A \in A_{\gamma} \cap A_{\gamma^{\prime}}$ such that $\alpha \in A \nRightarrow \beta \in A$. Since $A_{\gamma} \cap A_{\gamma^{\prime}}=r$, we know that $A \in r$ and this contradicts (4).

We turn our attention to the second parameter of the neighborhoods. Indeed, we can now restrict our attention to $C_{0}$ and $C_{1}$. Choose $\gamma \in C_{0}$. Choose $\gamma^{\prime} \in C_{1}$ such that $\left\{\gamma, \gamma^{\prime}\right\} \notin B_{\gamma}$. Since $\left|B_{\gamma}\right| \leq \aleph_{1}$ and $\left|C_{1}\right|=\kappa \geq \aleph_{2}$, this is possible. We claim that $\left\{\left(\gamma, \varnothing, B_{\gamma}\right),\left(\gamma^{\prime}, \varnothing, B_{\gamma^{\prime}}\right)\right\} \in U_{\varnothing, B_{\gamma}}(\gamma) \cap U_{\varnothing, B_{\gamma^{\prime}}}(\gamma)$. The only thing to check is that this point exists. Actually it may not be an element of $X_{2}$ but it is an element of $X_{1}$. To see this, we have to show that it is not true that $\left\{\gamma, \gamma^{\prime}\right\} \in B_{\gamma} \cap B_{\gamma^{\prime}}$. This is true simply because $\left\{\gamma, \gamma^{\prime}\right\} \notin B_{\gamma}$. This completes the proof that, if $\kappa$ is a regular cardinal greater than $\omega_{1}$, then $X_{1}$ is not $\aleph_{1}$-collectionwise normal with respect to discrete sets of cardinality $\aleph_{2}$.

We now assume $\kappa \geq \aleph_{3}$. Let $\left\{\gamma_{\delta}^{i}: \delta \in \omega_{3}, i \in 2\right\}$ list distinct elements so that each $\gamma_{\delta}^{i} \in C_{i}$. Define a mapping $f: \omega_{3} \rightarrow\left[\omega_{3}\right] \leq \omega_{1}$ by

$$
f(\delta)=\left\{\eta \in \omega_{3}:(\exists i, j \in 2)\left\{\gamma_{\delta}^{i}, \gamma_{\eta}^{i}\right\} \in B_{\gamma_{\delta}^{j}}\right\} .
$$

By Hajnal's free set lemma (see Theorem 3.1 .1 of [35]), there is a free set $F \in\left[\omega_{3}\right]^{\omega_{3}}$ for $f$. That is, such that $\delta, \delta^{\prime} \in F, \delta \neq \delta^{\prime} \Rightarrow \delta^{\prime} \notin f(\delta)$. Choose distinct $\delta_{0}, \delta_{1} \in F$. Let $\gamma_{i}=\gamma_{\delta_{i}}^{i}$. We claim that $\left\{\left(\gamma_{0}, \varnothing, B_{\gamma_{0}}\right),\left(\gamma_{1}, \varnothing, B_{\gamma_{1}}\right)\right\} \in$ $U_{\varnothing, B_{\gamma_{0}}}\left(\gamma_{0}\right) \cap U_{\varnothing, B_{\gamma_{1}}}\left(\gamma_{1}\right)$. The only thing to check is that this point exists as an element of $X_{2}$. To see this, we have to show that it is not true that $\left\{\gamma_{0}, \gamma_{1}\right\} \in$ $B_{\gamma_{0}} \cup B_{\gamma_{1}}$. Suppose, without loss of generality, that $\left\{\gamma_{0}, \gamma_{1}\right\} \in B_{\gamma_{0}}$. That is, $\left\{\gamma_{\delta_{0}}^{0}, \gamma_{\delta_{1}}^{1}\right\} \in B_{\gamma_{\delta_{0}}^{0}}$. By the definition of $f$, this means that $\delta_{1} \in f\left(\delta_{0}\right)$, which is a contradiction.

Finally we show that $X_{1}$ is not a covering $P_{\omega}$-space. Otherwise, there would be a family $\left\{A_{\alpha}, B_{\alpha}: \alpha \in \kappa\right\}$ such that each $U_{A_{\alpha}, B_{\alpha}}(\alpha)$ is a neighborhood of $\alpha$ and such that any countably many elements of $\left\{U_{A_{\alpha}, B_{\alpha}}(\alpha): \alpha \in \kappa\right\}$ are a locally 
finite family. We proceed as in the proof that $X_{1}$ fails to be $\aleph_{1}$-collectionwise normal with respect to discrete sets of cardinality $\aleph_{2}$ up to the decoding of normality. We choose $\left\{\gamma_{n}^{i}: n \in \omega\right\} \subset C_{i}$ for each $i \in \omega$. Let $B=\bigcup\left\{B_{\gamma_{n}^{i}}\right.$ : $i, n \in \omega\}$. Now $|B| \leq \aleph_{1}$. Choose $\alpha \in C_{\omega}-B$. This is possible since $\left|C_{\omega}\right|=$ $\aleph_{2}$. We claim that $\alpha$ is in the closure of the family $\left\{U_{A_{\gamma_{n}^{i}}, B_{\gamma_{n}^{i}}}\left(\gamma_{n}^{i}\right): i, n \in \omega\right\}$ of open sets. Suppose, otherwise, that $\alpha$ has a neighborhood $U_{A, B}(\alpha)$ which misses all but finitely many of these open sets. Since $\left\{r_{i}-r: i \in \omega\right\}$ is a disjoint family, we can choose $k \in \omega$ such that $\left(r_{k}-r\right) \cap A=\varnothing$. Since $\left\{A_{\gamma_{n}^{k}}-r_{k}: n \in \omega\right\}$ is a disjoint family, we can choose $p \in \omega$ such that $\left(A_{\gamma_{p}^{k}}-r_{k}\right) \cap A=\varnothing$. This means that $A_{\gamma_{p}^{k}} \cap A \subset r$. We claim that $U_{A, B}(\alpha) \cap U_{A_{\gamma_{p}^{k}}, B_{\gamma_{p}^{k}}}\left(\gamma_{p}^{k}\right) \neq \varnothing$. The isolated point $\left\{(\alpha, A, B),\left(\gamma_{p}^{k}, A_{\gamma_{p}^{k}}, B_{\gamma_{p}^{k}}\right)\right\}$ is in the intersection. If this isolated point did not exist, the reason could be, first, that there is $D \in A \cap A_{\gamma_{p}^{k}} \subset r$ such that $\alpha \in D$ is not equivalent to $\gamma_{p}^{k} \in D$. This contradicts step (4) in the thinning-out argument on p. 5. Another reason could be that $\left\{\alpha, \gamma_{p}^{k}\right\} \in B \cap B_{\gamma_{p}^{k}}$. We chose $\alpha \notin B_{\gamma_{p}^{k}}$ and so this does not happen.

Next, a collectionwise Hausdorff space which is not collectionwise normal. The space $X_{\mathrm{CWH}}$ is an example of an AND/AND construction. Of course, our example has character $2^{\aleph_{1}}$ which means that it provides no substitute for Fleissner's George [11].

Example 5. Let $F=Z \times \omega_{1}$ where $Z$ is an uncountable locally countable strongly zero-dimensional countably compact normal space and $\omega_{1}$ has the discrete topology. Let $\mathrm{BO}(F)$ be a base for $F$. Let $\mathrm{CWH}$ be the set of all discrete families of clopen countable sets in $F$. Let $J$ be the set of all $\{(A, P, Y),(B, Q, Z)\} \in\left[\mathrm{CO}(F) \times[\mathrm{CO}(F)]^{<\omega} \times[\mathrm{CWH}]^{<\omega}\right]^{2}$ which satisfy

$(C \in P \cap Q) \Rightarrow(A \subset C \Leftrightarrow B \subset C)$.

$(K \in Y \cap Z) \Rightarrow|\{C \in K: C \cap(A \cup B) \neq \varnothing\}| \leq 1$.

Let $X_{\mathrm{CWH}}=F \cup J$. A topology is defined on $X_{\mathrm{CWH}}$ by declaring $J$ to be a set of isolated points and defining an open set for each $U \in \mathrm{BO}(F), R \in[\mathrm{CO}(F)]^{<\omega}$, $W \in[C W H]^{<\omega}$ by letting $U(R, W)=U \cup\{\{(A, P, Y),(B, Q, Z)\}: A \subset U$, $P \supset R, Y \supset W\}$.

Lemma 9. The space $X_{\mathrm{CWH}}$ is normal and collectionwise Hausdorff but fails to be collectionwise normal.

Proof. The proof that $X_{\mathrm{CWH}}$ is a $T_{1}$ topological space which induces the product topology on $F$ is similar to that in the proof of Lemma 2. We note that $Z$ in Example 2 has identical separation properties to the space $X_{\mathrm{CWH}}^{+}$which is defined by removing the second condition from the definition of $J$. The only difference is that each isolated point has been replaced by a copy of $\left[[\mathrm{CWH}]^{<\omega}\right]^{2}$. This means that since $X_{\mathrm{CWH}}$ is a closed subspace of $X_{\mathrm{CWH}}^{+}, X_{\mathrm{CWH}}$ is also normal.

We now show that $X_{\mathrm{CWH}}$ is collectionwise Hausdorff. Let $D$ be a closed discrete subset of $X_{\mathrm{CWH}}$. We can assume that $D \subset F=Z \times \omega_{1}$. We can use the fact that $Z$ is countably compact to find a discrete family $K \in \mathrm{CWH}$ of basic clopen countable subsets of $F$ which separate $D$ in $F$. We claim that $\{U(\varnothing,\{K\}): U \in K\}$ separates $D$. Suppose that $\{(A, P, Y),(B, Q, Z)\} \in$ $U(\varnothing,\{K\}) \cap V(\varnothing,\{K\})$ where $U, V \in K$. Now $K \in Y \cap Z$ while $A \subset U$ and 
$B \subset V$. The requirement on $J$ implies that at most one element of $K$ intersects $A \cup B$. Now $U, V \in K$ and that is a contradiction. To show that $X_{\mathrm{CWH}}$ fails to be collectionwise normal, we will first inductively find $\left\{U_{\alpha}\left(R_{\alpha}, W_{\alpha}\right): \alpha \in \omega_{1}\right\}$ such that each $U_{\alpha}$ is a subset of a distinct copy of $Z$ and such that $\bigcup \bigcup\left\{W_{\beta}\right.$ : $\beta<\alpha\} \cap U_{\alpha}=\varnothing$. The "decoding" of collectionwise Hausdorff is finished. What remains is a standard normality "decoding" argument.

\section{A PARA-LINDELÖF SPACE}

Let us now turn to coding the para-Lindelöf property. Navy's space [20] was constructed by coding the $\sigma$-para-Lindelöf property and the property of normality. In our example we code para-Lindelöf directly. Unfortunately this would mean that we would not get even a Hausdorff space so we have to code regularity. We will code para-Lindelöf with an AND construction and regularity with an OR construction thus obtaining an OR/AND hybrid.

\section{Example 6. Let}

$F$ be an ultraparacompact space

$\mathrm{BO}(F)$ be a base of nonempty clopen subsets of $F$

PAR be a family of partitions of $F$

$t_{0} \in$ PAR to be fixed

$s: \mathrm{BO}(F) \rightarrow t_{0}$

REF $:$ PAR $\rightarrow$ PAR

$\pi: \mathrm{PAR} \times \mathrm{BO}(F) \rightarrow[\mathrm{BO}(F)]^{\omega}$ be a partial function

such that

$$
\begin{aligned}
& (\forall b \in \mathrm{BO}(F)) b \subset s(b) \in t_{0} \\
& (\forall t \in \mathrm{PAR}) t \subset \mathrm{BO}(F) \\
& (\forall \mathscr{U} \text { an open cover of } F)(\exists t \in \mathrm{PAR}): t \text { refines } \mathscr{U} \\
& (\forall t \in \operatorname{PAR}) \operatorname{REF}(t) \text { refines } t \\
& \operatorname{dom}(\pi)=\{(t, v) \in \operatorname{PAR} \times \mathrm{BO}(F): v \in \operatorname{REF}(t)\} \\
& (\forall t \in \operatorname{PAR})(\forall v \in \operatorname{REF}(t)) \pi(t, v) \in[t]^{\omega}
\end{aligned}
$$

Let $G$ be the set of $\left\{\left(b_{i}, P_{i}, s_{i}\right): i \in 2\right\} \in\left[\mathrm{BO}(F) \times[\mathrm{PAR}]^{<\omega} \times\left[\left[t_{0}\right]^{2}\right]^{<\omega}\right]^{2}$ which satisfy:

$$
\begin{aligned}
& t \in P_{0} \cap P_{1} \Rightarrow(\forall i \in 2)\left(b_{i} \subset v \in \operatorname{REF}(t) \Rightarrow b_{1-i} \cap \bigcup \pi(t, v) \neq \varnothing\right) \\
& \left\{s\left(b_{1}\right), s\left(b_{0}\right)\right\} \not \subset s_{0} \cup s_{1}, s\left(b_{0}\right) \neq s\left(b_{1}\right)
\end{aligned}
$$

Topologize $X=X\left(F, \mathrm{BO}(F)\right.$, PAR, $\left.t_{0}, s, \mathrm{REF}, \pi\right)=F \cup G$ by letting $G$ be a set of isolated points and letting

$$
U_{Q, s}(\sigma)=\sigma \cup\left\{\left\{\left(b_{i}, P_{i}, s_{i}\right): i \in 2\right\}: b_{1} \subset \sigma, P_{1} \supset Q, s_{1} \supset s\right\}
$$

for each $\sigma \in \mathrm{BO}(F), Q \in[\mathrm{PAR}]^{<\omega}, s \in\left[\left[t_{0}\right]^{2}\right]^{<\omega}$.

Note that it is an OR coding of regularity we have used not because of its logical appearance but because either $s_{i}$ can cause the neighborhoods to be disjoint. It all depends on whether there is a restriction on elements of the intersection or the union of the corresponding coordinates. Compare the proof of Corollary 10 for the use of this coding of regularity to obtain local collectionwise normality.

Lemma 10. The topological space $X$ is a zero-dimensional para-Lindelöf Hausdorff space. 
Proof. This is a topology because of

$$
U_{Q \cup Q^{\prime}, s \cup s^{\prime}}\left(\sigma \cap \sigma^{\prime}\right) \subset U_{Q, s}(\sigma) \cap U_{Q^{\prime}, s^{\prime}}\left(\sigma^{\prime}\right) .
$$

The subspace topology on $F$ is just its original topology. The fact that the points of $X$ are closed follows from $\bigcap\left\{U_{Q, s}(\sigma): s \in\left[\left[t_{0}\right]^{2}\right]^{<\omega}\right\}=\sigma$.

To see that the basic open sets are clopen, suppose that $x \in F-\sigma$. There must exist $\tau \in \mathrm{BO}(F)$ such that $\tau \cap \sigma=\varnothing$ and $x \in \tau$. We claim that $U_{\varnothing,\{s(\sigma), s(\tau)\}}(\tau) \cup U_{\varnothing, \varnothing}(\sigma)=\varnothing$. To see this, suppose that $\left\{\left(b_{i}, P_{i}, s_{i}\right): i \in 2\right\}$ were in the intersection. We can assume $b_{1} \subset \tau, b_{0} \subset \sigma$ and $s_{1} \supset\{s(\sigma), s(\tau)\}$. However $s\left(b_{0}\right)=s(\sigma)$ and $s\left(b_{1}\right)=s(\tau)$ yield a contradiction.

To see that $X$ is para-Lindelöf, it suffices to show that each $t \in$ PAR is the restriction of a locally countable open family in $X$ to $F$. We claim that $\left\{U_{\{t\}, \varnothing}(u): u \in t\right\}$ is as required. Fix $x \in F$ and $x \in v \in \operatorname{REF}(t)$. We shall show that $U_{\{t\}, \varnothing}(v) \cap U_{\{t\}, \varnothing}(u) \neq \varnothing \Rightarrow v \subset u \vee u \in \pi(t, v)$. This suffices since, for each $v$, there are countably many such $u$. Suppose $\left\{\left(b_{i}, P_{i}, s_{i}\right): i \in 2\right\}$ is in the intersection. We can assume that $v \supset b_{1}, u \supset b_{0}$ (unless $v \subset u$ ) and $t \in P_{0} \cap P_{1}$. Now $v \in \operatorname{REF}(t)$ which implies $b_{0} \cap \bigcup \pi(t, v) \neq \varnothing$. Finally $\pi(t, v) \subset t$ and $b_{0} \subset u \in t$ implies $u \in \pi(t, v)$. Of course, each isolated point is an element of at most two elements of the family.

Definition 3. Let $\kappa$ be an infinite cardinal. If $\sigma$ is a partial function from $\omega$ to $\kappa$, we use $[\sigma]$ to denote $\left\{f \in \kappa^{\omega}: f \supset \sigma\right\}$. Let $\psi: \kappa \rightarrow[\kappa]^{\omega}$.

Let $F$ be the metric space $\kappa^{\omega}$.

Let $\mathrm{BO}(F)=\left\{[\sigma]:(\exists n \geq 1) \sigma \in \kappa^{n}\right\}$.

Let $t_{0}=\{[(0, \alpha)]: \alpha \in \kappa\}$.

Let $[\sigma] \in \mathrm{BO}(F) \Rightarrow s([\sigma])=[(0, \sigma(0))] \in t_{0}$.

Let $t \in \operatorname{PAR} \Rightarrow \operatorname{REF}(t)=\{[\sigma \cup\{(\operatorname{dom}(\sigma), \alpha)\}]: \sigma \in t, \alpha \in \kappa\}$.

Let PAR be the family of all partitions of $F$ into elements of $\mathrm{BO}(F)$.

Let $\pi(t,[\sigma \cup\{(\operatorname{dom}(\sigma), \alpha)\}])=\left\{t_{\beta}: \beta \in \psi(\alpha)\right\}$ for each $t=\left\{t_{\beta}: \beta \in\right.$ $\kappa\} \in \operatorname{PAR}$ and $[\sigma] \in t$ and $\alpha \in \kappa$.

We denote the space $X\left(F, \mathrm{BO}(F), \mathrm{PAR}, t_{0}, s, \mathrm{REF}, \pi\right)$ by $X(\psi)$.

We need some definitions.

Definition $4[9,15]$. If $L \subset \mathrm{BO}(F)$ and $\mathscr{F}$ is a filter on $\kappa$, then we say that $L$ is $n-\mathscr{F}$-full if

$$
\begin{aligned}
& (\forall[\sigma] \in L) \operatorname{dom}(\sigma)=n . \\
& (\forall[\sigma] \in L)(\forall i<n)\{\alpha \in \kappa:(\forall \tau \in L) \tau \not \supset \sigma \mid i \cup\{(i, \alpha)\}\} \notin \mathscr{F} .
\end{aligned}
$$

If $\mathscr{F}$ is the filter of all sets whose complements have cardinality less than $\kappa$, then we say that $L$ is $n$-full.

Definition 5. If $L \subset \mathrm{BO}(F)$ is $n$-full and $\{Q(\sigma):[\sigma] \in L\}$ is a family of sets, then we say that $\{Q(\sigma):[\sigma] \in L\}$ is an $n$-full $\Delta$-system if there is a family of sets $\left\{\Delta(\sigma\lceil i):[\sigma] \in L, i<n\}\right.$ such that $\left[\sigma_{0}\right],\left[\sigma_{1}\right] \in L$ and $\sigma_{0}\left\lceil i=\sigma_{1}\lceil i=\sigma\right.$ and $\sigma_{0}(i) \neq \sigma_{1}(i)$ implies that $Q\left(\sigma_{0}\right) \cap Q\left(\sigma_{1}\right)=\Delta(\sigma)$.

Definition 6. If $L \subset \mathrm{BO}(F)$ is $n$-full and $\left\{s^{*}(\sigma):[\sigma] \in L\right\}$ is a family of subsets of $t_{0}$, then we say that $\left\{s^{*}(\sigma):[\sigma] \in L\right\}$ is free if $\left[\sigma_{0}\right],\left[\sigma_{1}\right] \in L$ and $\sigma_{0}(0) \neq \sigma_{1}(0)$ implies that $\left[\left(0, \sigma_{0}(0)\right)\right] \notin s^{*}\left(\sigma_{1}\right)$ and $\left[\left(0, \sigma_{1}(0)\right)\right] \notin s^{*}\left(\sigma_{0}\right)$.

Lemma 11. If $\kappa$ is a regular uncountable cardinal and $\mathscr{F}$ is a normal filter on $\kappa$ (actually any countably complete filter on which we can do the $\Delta$-system 
lemma is sufficient) and $\mathscr{U}$ is any open cover of $X(\psi)$, then there is an $n-\mathscr{F}$. full set $L \subset \mathrm{BO}(F)$ and an open refinement $\left\{U_{Q(\sigma), s(\sigma)}([\sigma]):[\sigma] \in L\right\}$ of $\mathscr{U}$ such that $\{\bigcup s(\sigma):[\sigma] \in L\}$ is free (and an $n$-full $\Delta$-system with respect to $\left.\left\{\Delta^{\prime}(\sigma \mid i):[\sigma] \in L, i<n\right\}\right)$ and $\{Q(\sigma):[\sigma] \in L\}$ is an $n$-full $\Delta$-system with respect to $\{\Delta(\sigma \mid i):[\sigma] \in L, i<n\}$. Furthermore, for each $t \in \Delta(\varnothing)$, $\left[\sigma_{0}\right],\left[\sigma_{1}\right] \in L$, we have $\left(\exists s^{0} \in t\right) \sigma_{0} \subset s^{0} \Rightarrow\left(\exists s^{1} \in t\right) \sigma_{1} \subset s^{1} \wedge \operatorname{dom}\left(s^{0}\right)=$ $\operatorname{dom}\left(s^{1}\right)$.

Proof. First find $L_{0} \in \mathrm{BO}(F)$ which partitions $F$ such that, for all $[\sigma] \in L_{0}$, there is an open set $U_{Q(\sigma), s(\sigma)}([\sigma])$ which is contained in an element of $\mathscr{U}$. To do this, for each $f \in F$, there is an element of $\mathrm{BO}(F)$ which contains $f$ and which is the intersection with $F$ of some basic open set in $X$ which is contained in an element of $\mathscr{U}$. Consider the family of such elements of $\mathrm{BO}(F)$ of minimal domain. These partition $F$ since any two elements of $\mathrm{BO}(F)$ are either disjoint or else one is contained in the other.

Next we find an $n-\mathscr{F}$-full subset $L_{1}$ of $L_{0}$. We do this by marking each element of $\mathrm{BO}(F) \cup\{F\}$ with an integer. An element of $L_{0}$ is marked with a 0 . Now if an element of $\mathrm{BO}(F) \cup\{F\}$ has measure 0 many successors which are marked with a 0 , then those successors are removed. All remaining predecessors of elements marked with a 0 are marked with a 1 . If an element of $\mathrm{BO}(F) \cup\{F\}$ has measure 0 many successors marked with a 1 , then those successors are removed. All remaining predecessors of elements marked with a 1 are marked with a 2. This process is continued in the obvious fashion. When the process is complete, the number of successors removed from any element is only the union of countably many sets of measure 0 . Thus we can trace a branch through the remaining tree and find an element of $L_{0}$ at level $n$. We now construct $L_{1}$ by taking all elements at level $n$ which remain and are marked with a 0 .

$L_{1}$ is $n-\mathscr{F}$-full since each element at level $i<n$ which remains and is marked with an $n-i$ has positive measure many successors at level $i+1$ which are marked with an $n-(i+1)$.

Next, we find an $L_{2}$ so that $\left\{\bigcup s(\sigma):[\sigma] \in L_{2}\right\}$ and $\left\{Q(\sigma):[\sigma] \in L_{2}\right\}$ are $n$-full $\Delta$-systems. We shall describe the second case since the first case is similar. We do this by defining $\left\{Q(\sigma \mid i):[\sigma] \in L_{1}, i<n\right\}$ and removing certain vertices. For each vertex $\tau=\sigma\lceil(n-1)$ at height $n-1$, remove enough vertices $\sigma$ at height $n$ so that the $\{Q(\sigma): \sigma \mid(n-1)=\tau\}$ is a $\Delta$-system. Call the root of this $\Delta$-system $Q(\tau)$. Now for each vertex $v$ at height $n-2$, remove enough vertices at height $n-1$ (and their successors) so that the $Q(w)$ 's for successors $w$ of $v$ are a $\Delta$-system. Continue this process down to level 0 .

Finally, we must get $\{\bigcup s(\sigma):[\sigma] \in L\}$ to be free. Just remove $\Delta^{\prime}(\varnothing)$ from the first branching.

Lemma 12. If $U_{Q, s}([\sigma]) \cap U_{Q^{\prime}, s^{\prime}}\left(\left[\sigma^{\prime}\right]\right)=\varnothing$ and $\sigma(0) \neq \sigma^{\prime}(0)$ and $s\left(\sigma^{\prime}\right) \notin \bigcup s$ and $s(\sigma) \notin U s^{\prime}$, then $U_{Q \cap Q^{\prime}, \varnothing}([\sigma]) \cap U_{Q \cap Q^{\prime}, \varnothing}\left(\left[\sigma^{\prime}\right]\right)=\varnothing$.

Proof. Suppose that $\left\{\left(b_{i}, P_{i}, s_{i}\right): i \in 2\right\}$ is an element of $U_{Q^{\prime} Q^{\prime}, \varnothing}([\sigma]) \cap$ $U_{Q \cap Q^{\prime}, \varnothing}\left(\left[\sigma^{\prime}\right]\right)=\varnothing$. We assume that $b_{0} \subset[\sigma]$ and $b_{1} \subset\left[\sigma^{\prime}\right]$. We claim that $\left\{\left(b_{0}, Q, s\right),\left(b_{1}, Q^{\prime}, s^{\prime}\right)\right\} \in U_{Q, s}([\sigma]) \cap U_{Q^{\prime}, s^{\prime}}\left(\left[\sigma^{\prime}\right]\right)=\varnothing$ which would be a contradiction. The only way this would not happen is if that point $\left\{\left(b_{0}, Q, s\right)\right.$, $\left.\left(b_{1}, Q^{\prime}, s^{\prime}\right)\right\}$ did not exist. If this is because of $t \in Q \cap Q^{\prime}$, then since $t \in P_{0} \cap P_{1},\left\{\left(b_{i}, P_{i}, s_{i}\right): i \in 2\right\}$ would also not exist. The other reason could be that $\left\{s\left(b_{0}\right), s\left(b_{1}\right)\right\} \in s \cup s^{\prime}$ or $s\left(b_{0}\right)=s\left(b_{1}\right)$. Since $s\left(b_{0}\right)=[0, \sigma(0)]=s(\sigma)$ and $s\left(b_{1}\right)=\left[0, \sigma^{\prime}(0)\right]=s\left(\sigma^{\prime}\right)$, this cannot happen. 
Lemma 13. Suppose $\psi: \omega_{1} \rightarrow\left[\omega_{1}\right]^{\omega}$ is defined by $\psi(\alpha)=\alpha . X(\psi)$ fails to be collectionwise normal.

Proof. We shall show that $t_{0}$ is an unseparated discrete family of closed sets in $X$. Let $\mathscr{U}$ be an open cover of $X$ which separates $t_{0}$. Use $\mathscr{F}$ the filter of closed unbounded subsets of $\omega_{1}$. Apply Lemma 11 to get an $n-\mathscr{F}$-full $L$ and $n$-full $\Delta$-system $\{Q(\sigma):[\sigma] \in L\}$ and free $\{\bigcup s(\sigma):[\sigma] \in L\}$. We will find $\left[\sigma_{0}\right],\left[\sigma_{1}\right] \in L$ such that $\sigma_{0}(0) \neq \sigma_{1}(0)$ and $U_{Q\left(\sigma_{0}\right), t\left(\sigma_{0}\right)}\left(\left[\sigma_{0}\right]\right) \cap U_{Q\left(\sigma_{1}\right), t\left(\sigma_{1}\right)}\left(\left[\sigma_{1}\right]\right) \neq$ $\varnothing$. By Lemma 12 , we need only show that $U_{\Delta(\varnothing), \varnothing}\left(\left[\sigma_{0}\right]\right) \cap U_{\Delta(\varnothing), \varnothing}\left(\left[\sigma_{1}\right]\right) \neq$ $\varnothing$. Otherwise $\left\{\left(\left[\sigma_{0}\right], \Delta(\varnothing), \varnothing\right),\left(\left[\sigma_{1}\right], \Delta(\varnothing), \varnothing\right)\right\}$ is not in the intersection. This means that $\exists t \in \Delta(\varnothing)$ and $i \in 2$ such that $\left[\sigma_{i}\right] \subset v \in \operatorname{REF}(t)$ and yet $\left[\sigma_{1-i}\right] \cap \bigcup \pi(t, v)=\varnothing$. Suppose $v=\sigma \cup\{(j, \alpha)\}$ where $[\sigma] \in t$ and $\operatorname{dom}(\sigma)=j$. Since $\left[\sigma_{i}\right] \subset v$, we know that $v=\left[\sigma_{i} \mid(j+1)\right]$ and also $\sigma_{i}(j)=\alpha$. If $\operatorname{dom}\left(\sigma_{i}\right)=n$, we get that $j<n$. Now since $\sigma_{i}$ is contained in some element of $t$ (namely $\sigma$ ) of domain $j$, we know that $\sigma_{1-i}$ is contained in some element $t_{\beta}$ of $t$ such that $\operatorname{dom}\left(t_{\beta}\right)=j$. This means that $t_{\beta} \supset\left[\sigma_{1-i}\lceil j]\right.$. Since we are assuming that $\left[\sigma_{1-i}\right] \cap \bigcup \pi(t, v)=\varnothing$, we know that $t_{\beta} \notin \pi(t, v)$. This means that $\beta \notin \psi(\alpha)$. Since $\alpha=v(j)=\sigma_{i}(j)$ and, in a finite inductive construction of $\sigma_{0}, \sigma_{1}$, we were free to choose $\sigma_{i}(j)$ in an uncountable set, we deduce that we could always arrange that $\beta \in \psi(\alpha)$ which is a contradiction.

Corollary 1 (Navy). There is a para-Lindelöf regular space which is not collectionwise normal.

Note that this space has character $2^{w(F)}$ which means that it provides no substitute for Navy's spaces [20]. It just illustrates that the coding of normality into Fleissner's space with a $\sigma$-locally countable base is not the trick that is needed to get para-Lindelöf. You just have to code all instances of para-Lindelöf, not just the "level" ones.

Problem 3. Does the existence of a nonmetrizable normal Moore space imply the existence of a para-Lindelöf nonmetrizable normal Moore space?

The normal Moore space problem enjoyed lots of consistent counterexamples long before para-Lindelöf raised its head. However Caryn Navy [20] managed to show how to use $\mathrm{MA}+\neg \mathrm{CH}$ to get a para-Lindelöf example of a nonmetrizable normal Moore space. Fleissner's CH example of a nonmetrizable normal Moore space [13] turns out to be para-Lindelöf (after all, he was modifying Navy's example). The only part that is not clear is whether the singular cardinals hypothesis can get you a para-Lindelöf counterexample to the normal Moore space conjecture. Probably the best positive result that can be hoped for is to show that Fleissner's SCH counterexample can be modified to be para-Lindelöf. That would be a good result since a deep understanding of Fleissner's space would be required and that space does have to be digested. A negative result is more likely and would really illustrate the difference between Fleissner's $\mathrm{CH}$ example and his $\mathrm{SCH}$ example $[13,10]$ and that would be valuable.

Problem 4. Are para-Lindelöf regular spaces countably paracompact?

This is the main open problem on para-Lindelöf spaces. The original question was whether para-Lindelöf was equivalent to paracompact-one more feather in the cap of equivalences of paracompactness established by Stone and Michael 
in the 1950s (see Burke's article in the handbook [4]). This question was finally solved by Caryn Navy, a student of Mary Ellen Rudin, in 1981 [20]. Her construction was a rather general one that permitted quite a lot of latitude; she obtained first countable ones under $\mathrm{MA}+\neg \mathrm{CH}$ using the Moore plane, she obtained a ZFC example using Bing's space. Fleissner [13, 10] later modified this example to be a Moore space under the continuum hypothesis, thus solving the normal Moore space conjecture. Certain properties seemed hard to get however. These difficulties each gave rise to questions which were listed in Navy's thesis. Problem 4 is due to the fact that all the constructions are intrinsically countably paracompact. I tried for a long time to build in the failure of countable paracompactness but each time para-Lindelöf failed as well. It may be useful to note that the whole idea of Navy's construction was to take Fleissner's space of 1979 [9] which was $\sigma$-para-Lindelöf but not paracompact and build in a way to "separate" the countably many locally countable families so that one locally countable refinement is obtained. This way was normality. The construction in this section provides another way to get a para-Lindelöf space but I still do not see how to make it fail to be countably paracompact. A possible way of getting a para-Lindelöf space which is not countably paracompact may be to iterate a normal para-Lindelöf space which is not collectionwise normal in an $\omega$-sequence (see [33]) and get a Dowker space.

Problem 5. Is there a para-Lindelöf Dowker space?

Another question which I think is important is

Problem 6. Are para-Lindelöf collectionwise normal spaces paracompact?

This was first asked by Fleissner and Reed in 1977 [15].

\section{AN UNSEPARATED FAMILY OF COPIES OF $[0,1]$}

This section studies more closely the structure of collectionwise Hausdorff normal spaces which are not collectionwise normal. Fifty years of effort towards solving the normal Moore space problem led in 1974 to the result that the solution was to be found in the construction, or the proof that it is impossible, of normal collectionwise Hausdorff Moore spaces which are not collectionwise normal. At that time, no examples of normal collectionwise Hausdorff spaces which are not collectionwise normal had been constructed, much less Moore examples. In the decade that followed, two basic examples of such spaces were constructed. In 1975, Fleissner [11] constructed a space which he called "George" which was normal and collectionwise Hausdorff but which contained an unseparated family of copies of the ordinal space $\omega_{1}$. In 1981, Navy [20] constructed a normal collectionwise Hausdorff space with an unseparated family of nonseparable metric spaces of weight $\aleph_{1}$. These two constructions provide the "prototypes" of normal collectionwise Hausdorff spaces which are not collectionwise normal. The latter prototype proved more successful and led to Fleissner's solution [13, 10] of the normal Moore space problem in 1982. Whenever there are only a small number of examples of a certain kind, many questions arise by examining the special nature of those examples.

Problem 7. Characterize those spaces $X$ for which there exists a normal collectionwise Hausdorff space which is not collectionwise normal with respect to copies of $X$. 
While this general question was not posed until 1985, specific forms of this question were studied as early as 1979. For example, Gruenhage showed in 1979 [16] that under $\mathrm{MA}+\neg \mathrm{CH}$, perfectly normal locally compact spaces which are collectionwise normal with respect to compact sets are paracompact. This hypothesis "collectionwise normal with respect to compact sets" led naturally to the question "Are collectionwise Hausdorff (normal) spaces always collectionwise normal with respect to compact sets"? Balogh improved Gruenhage's result in 1982 [1] by weakening the hypothesis of collectionwise normal with respect to compact sets to that of collectionwise Hausdorff but the question remained. This question turned out to be difficult enough even without requiring normality. In 1980, in an unpublished result, Fleissner and Reed showed that, if there is a measurable cardinal, then there is a regular collectionwise Hausdorff space which is not collectionwise normal with respect to compact sets. A few years later, Reed [23] constructed such a space in ZFC and got it to be first countable as well. Mary Ellen Rudin constructed (independently of and shortly before the results of this paper) a normal collectionwise Hausdorff space which is not collectionwise normal with respect to copies of $2^{\omega}$ under the assumption of $\diamond$ but the question remained open in ZFC.

In this section, we solve this question by constructing a normal collectionwise Hausdorff space which is not collectionwise normal with respect to copies of $[0,1]$. The method which is used to construct this space is applicable, without complications, to many other underlying sets and can, therefore, be used to construct many other counterexamples.

We do this by coding normality with an AND construction and collectionwise Hausdorff with an AND construction. The ideas will be described in a general setting. To simplify the proof, we will assume that the underlying space is strongly zero-dimensional. This assumption will be complemented by a lemma which enables us to go to any space from a closed strongly zero-dimensional subspace of suitable complexity.

Example 7. Let $Z$ be a normal collectionwise Hausdorff space which is strongly zero-dimensional. Let $D$ be the family of closed discrete subsets of $Z$. Suppose $B$ is a family of clopen subsets of $Z$ and $m: B \rightarrow[0,1]$ is a function such that

1. $(\forall d \in D)(\forall \varepsilon>0)(\exists U \in B): d \subset U \wedge m(U)<\varepsilon$.

2. $(\forall E \in[B] \leq \omega) \sum\{m(U): U \in E\}<1 \Leftrightarrow \bigcup E \neq Z$.

Let $\kappa$ be the least cardinal such that $|Z| \leq \kappa$ and $\kappa^{\omega}=\kappa$. Let $T={ }^{\kappa} D$. Let $\mathbb{N}^{T}$ have the $G_{\delta}$-product topology (that is, the basic open sets are determined by countable partial functions from $T$ into $\mathbb{N}$ ). Let $\pi: \kappa \rightarrow \mathbb{N}^{T}$ have dense range $(\pi$ exists by the Hewitt-Marczewski-Pondiczery theorem; see Theorem 2.3.15 of [7] or Lemma 1.4 of [5]). Let $\beta: T \times \kappa \rightarrow B$ be such that

$$
\begin{aligned}
& t(\alpha) \subset \beta((t, \alpha)), \\
& m(\beta((t, \alpha))) \cdot \pi(\alpha)(t)<1 .
\end{aligned}
$$

The function $\beta$ exists by (1). Let $Y=Z \times \kappa$ have the product topology where $\kappa$ has the discrete topology. Let $J$ be the set of

$$
\left\{(V, G, P),\left(V^{\prime}, G^{\prime}, P^{\prime}\right)\right\} \in\left[\mathrm{CO}(Y) \times[T]^{<\omega} \times[\mathrm{CO}(Y)]^{<\omega}\right]^{2}
$$


such that

$$
\begin{aligned}
& A \in P \cap P^{\prime} \Rightarrow\left(V \subset A \Leftrightarrow V^{\prime} \subset A\right), \\
& t \in G \cap G^{\prime} \Rightarrow V \cup V^{\prime} \not \subset \cup\{\beta(t, \alpha) \times\{\alpha\}: \alpha \in \kappa\}, \\
& V \text { and } V^{\prime} \text { are nonempty subsets of different copies of } Z .
\end{aligned}
$$

Topologize $X(Z, B, m)=Y \cup J$ by letting the elements of $J$ be isolated and letting a neighborhood $U(A, F)$ be defined for each nonempty $U \in \operatorname{CO}(Y)$, $A \in[\mathrm{CO}(Y)]^{<\omega}$, and $F \in[T]^{<\omega}$ by

$$
U(A, F)=U \cup\left\{\left\{(V, G, P),\left(V^{\prime}, G^{\prime}, P^{\prime}\right)\right\}: V \subset U, G \supset F, P \supset A\right\} .
$$

Lemma 14. $X(Z, B, m)$ is a normal collectionwise Hausdorff space which is not collectionwise normal with respect to copies of $Z$.

Proof. This assignment of basic open neighborhoods defines a space since

$$
U \subset U^{\prime}, A \supset A^{\prime}, F \supset F^{\prime} \Rightarrow U(A, F) \subset U^{\prime}\left(A^{\prime}, F^{\prime}\right)
$$

and so

$$
U(A, F) \cap U^{\prime}\left(A^{\prime}, F^{\prime}\right) \supset\left(U \cap U^{\prime}\right)\left(A \cup A^{\prime}, F \cup F^{\prime}\right) .
$$

Compare the third condition on $J$ with the coding of regularity in Example 6 and the coding of local collectionwise normality in Corollary 10. Since $U(A, F) \cap Y=U$ and $Y$ is zero-dimensional, the subspace topology on $Y$ is identical to its original topology. Since $\bigcap\left\{U(A, F): F \in[T]^{<\omega}\right\}=U$ and $Y$ is $T_{1}$, we know that $X(Z, B, m)$ is $T_{1}$. Note that this incorporation of Example 2 implies that $X$ is normal. To be explicit, suppose $K_{0}$ and $K_{1}$ are disjoint closed sets. Without loss of generality, these closed sets are subsets of $Y$ and, since $Y$ is strongly zero-dimensional and normal, we assume that $K_{0}$ and $K_{1}$ partition $Y$ and thus are clopen subsets of $Y$. We use the third coordinate only. We claim that

$$
U\left(\left\{K_{1}\right\}, \varnothing\right) \cap W\left(\left\{K_{1}\right\}, \varnothing\right)=\varnothing
$$

whenever $U \subset K_{1}$ and $W \cap K_{1}=\varnothing$. Suppose that $\left\{(V, G, P),\left(V^{\prime}, G^{\prime}, P^{\prime}\right)\right\}$ witnesses otherwise. Without loss of generality, $U \supset V$ and $W \supset V^{\prime}$. We know $P \supset\left\{K_{1}\right\}$ and $P^{\prime} \supset\left\{K_{1}\right\}$ and so that $K_{1} \in P \cap P^{\prime}$. This means that $V \subset K_{1}$ if and only if $V^{\prime} \subset K_{1}$. Now $K_{1} \supset U \supset V$ implies that $K_{1} \supset V^{\prime}$ and so, since $V^{\prime} \neq \varnothing$, we have $K_{1} \cap W \neq \varnothing$ which is a contradiction. Since $X(Z, B, m)$ is normal and $T_{1}$, it is also Hausdorff.

Let $A$ be a discrete family of points in $X$. Assume, without loss of generality, that $A \subset Y$. Let $t: \kappa \rightarrow D$ be such that $A=\bigcup\{t(\alpha) \times\{\alpha\}$ : $\alpha \in \kappa\}$. We assert that $\left\{U_{\alpha}(\varnothing,\{t\}): \alpha \in \kappa\right\}$ is a disjoint family where each $U_{\alpha}=\beta(t, \alpha) \times\{\alpha\} \supset t(\alpha) \times\{\alpha\}$. Suppose $\left\{(V, G, P),\left(V^{\prime}, G^{\prime}, P^{\prime}\right)\right\} \in$ $U_{\alpha}(\varnothing,\{t\}) \cap U_{\alpha^{\prime}}(\varnothing,\{t\})$. Without loss of generality $V \subset U_{\alpha}$ and $V^{\prime} \subset U_{\alpha^{\prime}}$. Since $t \in G \cap G^{\prime}$, we have $V \cup V^{\prime} \not \subset \bigcup\{\beta(t, \alpha) \times\{\alpha\}: \alpha \in \kappa\}$ which implies $U_{\alpha} \cup U_{\alpha^{\prime}} \not \subset \cup\{\beta(t, \alpha) \times\{\alpha\}: \alpha \in \kappa\}$ which is a contradiction. To finish the proof, we show that, for each $\alpha \in \kappa, t(\alpha) \times\{\alpha\}=\left\{\left(z_{\xi}, \alpha\right): \xi \in \zeta\right\}$ is separated. Since $Z$ is collectionwise Hausdorff and zero-dimensional, we know that there is a disjoint family $\left\{U_{\xi}: \xi<\zeta\right\}$ of clopen subsets of $Z$ such that $z_{\xi} \in U_{\xi}$. Now $\left\{\left(U_{\xi} \times\{\alpha\}\right)(\varnothing, \varnothing): \xi<\zeta\right\}$ is a disjoint family of open sets which separates $t(\alpha) \times\{\alpha\}$. We show that if $U$ and $W$ are disjoint subsets of the same copy of $Z$, then $U(\varnothing, \varnothing) \cap W(\varnothing, \varnothing)=\varnothing$. Suppose, otherwise that 
$\left\{(V, G, P),\left(V^{\prime}, G^{\prime}, P^{\prime}\right)\right\}$ is in the intersection. Thus $U \supset V$ and $W \supset V^{\prime}$ without loss of generality. Thus $V$ and $V^{\prime}$ are in the same copy of $Z$ and that is a contradiction to the definition of the isolated points.

We show that $X$ is not collectionwise normal. Suppose that $\left\{U_{\alpha}: \alpha \in \kappa\right\}$ is a disjoint family of open sets such that $U_{\alpha} \supset Z \times\{\alpha\}$. We will find, by induction,

basic open sets $\left\{V_{\gamma}\left(A_{\gamma}, F_{\gamma}\right): \gamma \in \omega_{1}\right\}$,

distinct ordinals $\left\{\alpha_{\gamma}: \gamma \in \omega_{1}\right\}$,

points $x_{\gamma}$,

such that

$$
\begin{aligned}
& x_{\gamma} \in V_{\gamma} \subset Z \times\left\{\alpha_{\gamma}\right\}, \\
& \gamma^{\prime}<\gamma<\omega_{1} \text { and } t \in F_{\gamma^{\prime}} \text { implies } x_{\gamma} \notin \beta\left(t, \alpha_{\gamma}\right) \times\left\{\alpha_{\gamma}\right\}, \\
& V_{\gamma}\left(A_{\gamma}, F_{\gamma}\right) \subset U_{\alpha_{\gamma}} .
\end{aligned}
$$

Having defined these basic open sets, we make

Claim. $V_{\gamma}\left(A_{\gamma}, \varnothing\right) \cap V_{\gamma^{\prime}}\left(A_{\gamma^{\prime}}, \varnothing\right)$ does not contain any $\left\{(V, \varnothing, P),\left(V^{\prime}, \varnothing, P^{\prime}\right)\right\}$ where $x_{\gamma} \in V$ and $x_{\gamma^{\prime}} \in V^{\prime}$.

Proof of claim. If such a point were in the intersection, then the point $\left\{\left(V, F_{\gamma}, P\right),\left(V^{\prime}, F_{\gamma^{\prime}}, P^{\prime}\right)\right\}$ would be in $V_{\gamma}\left(A_{\gamma}, F_{\gamma}\right) \cap V_{\gamma^{\prime}}\left(A_{\gamma^{\prime}}, F_{\gamma^{\prime}}\right)$ unless that point did not exist. Thus there must be $t \in F_{\gamma} \cap F_{\gamma^{\prime}}$ such that (assuming $\left.\gamma^{\prime}<\gamma\right) \quad x_{\gamma} \in V \subset \bigcup\{\beta(t, \alpha): \alpha \in \kappa\}$ and thus $x_{\gamma} \in \beta\left(t, \alpha_{\gamma}\right) \times\left\{\alpha_{\gamma}\right\}$ which is a contradiction. The claim is proved.

Now a standard $\Delta$-system argument can be used to show that the claim is false. Without loss of generality, assume $\left\{A_{\gamma}: \gamma \in \omega_{1}\right\}$ is a $\Delta$-system with root $\Delta$ and assume $x_{\gamma} \in U$ if and only if $x_{\gamma^{\prime}} \in U$ whenever $\gamma<\gamma^{\prime}<\omega_{1}$ and $U \in \Delta$. For each $\gamma$, choose $V_{\gamma}^{*} \subset V_{\gamma} \subset Z \times\left\{\alpha_{\gamma}\right\}$ which contains $x_{\gamma}$ and which lies on the appropriate side of each $U \in \Delta$. That is,

$$
(\forall U \in \Delta)\left(x_{\gamma} \in U \Rightarrow V_{\gamma}^{*} \subset U\right) \wedge\left(x_{\gamma} \notin U \Rightarrow V_{\gamma}^{*} \cap U=\varnothing\right) .
$$

We note that $\left\{\left(V_{\gamma}^{*}, \varnothing, A_{\gamma}\right),\left(V_{\gamma^{\prime}}^{*}, \varnothing, A_{\gamma^{\prime}}\right)\right\}$ does lie in the intersection. To check that this point exists, note that

$$
A \in A_{\gamma} \cap A_{\gamma^{\prime}} \Rightarrow A \in \Delta \Rightarrow\left(x_{\gamma} \in A \Leftrightarrow x_{\gamma^{\prime}} \in A\right) \Rightarrow\left(V_{\gamma}^{*} \subset A \Leftrightarrow V_{\gamma^{\prime}}^{*} \subset A\right) .
$$

To do the induction, suppose $\left\{V_{\eta}\left(A_{\eta}, F_{\eta}\right): \eta<\gamma\right\},\left\{\alpha_{\eta}: \eta<\gamma\right\}$, and $\left\{x_{\eta}: \eta<\right.$ $\gamma\}$ have been defined. Let $\left\{n_{\eta}: \eta<\gamma\right\} \subset \mathbb{N}$ be such that $\sum\left\{1 / n_{\eta}: \eta<\gamma\right\}<1$. Let $\delta: T \rightarrow \mathbb{N}$ be a partial function defined on the domain $\bigcup\left\{F_{\eta}: \eta<\gamma\right\}$ as follows. If $t \in \bigcup\left\{F_{\eta}: \eta<\gamma\right\}$, then let $\eta(t)$ be the minimal $\eta<\gamma$ such that $t \in F_{\eta(t)}$. Now define $\delta(t)=n_{\eta(t)} \cdot\left|F_{\eta(t)}\right|$. Now $|\operatorname{dom}(\delta)| \leq \omega$ implies that we can find $\alpha_{\gamma}$ distinct from each $\alpha_{\eta}$ for $\eta<\gamma$ such that $\pi\left(\alpha_{\gamma}\right) \supset \delta$ (since if there were only countably many such $\alpha_{\gamma}$ then we could diagonalize away from them to get a countable partial function with no extension). Find $x_{\gamma} \in Z \times\left\{\alpha_{\gamma}\right\}-\bigcup\left\{\beta\left(t, \alpha_{\gamma}\right) \times\left\{\alpha_{\gamma}\right\}: t \in \operatorname{dom}(\delta)\right\}$. This is possible since

$$
\begin{aligned}
& \sum\left\{m\left(\beta\left(\left(t, \alpha_{\gamma}\right)\right)\right): t \in \operatorname{dom}(\delta)\right\} \leq \sum\left\{1 / \pi\left(\alpha_{\gamma}\right)(t): t \in \operatorname{dom}(\delta)\right\} \\
& =\sum\left\{\sum\{1 / \delta(t): \eta(t)=\eta\}: \eta<\gamma\right\} \leq \sum\left\{1 / n_{\eta}: \eta<\gamma\right\}<1
\end{aligned}
$$

and so $\left\{\beta\left(t, \alpha_{\gamma}\right): t \in \operatorname{dom}(\delta)\right\}$ does not cover $Z$ by the condition on $m$. Find $V_{\gamma} \subset Z \times\left\{\alpha_{\gamma}\right\}$ such that $V_{\gamma}\left(A_{\gamma}, F_{\gamma}\right) \subset U_{\alpha_{\gamma}}$ and $x_{\gamma} \in V_{\gamma}$.

These arguments imply that $\{Z \times\{\alpha\}: \alpha \in \kappa\}$ is an unseparated discrete family of closed sets and the proof is complete. 
We continue with corollaries to the main theorem:

Corollary 2. There is a normal collectionwise Hausdorff space which is not collectionwise normal with respect to compact metric sets.

Proof. Let $Z=2^{\omega}, B=\mathrm{CO}(Z)$ and let $m$ be the product measure.

Corollary 3. There is a normal space which is collectionwise normal with respect to metrizable sets but is not collectionwise normal with respect to compact sets.

Proof. Let $Z$ be the modification of the Alexandroff double arrow space (see Exercise 3.10.C of [7]) which is obtained by starting with $2^{\omega}$ instead of $[0,1]$. Let $m$ be the product measure on $2^{\omega} \times 2$. Let $B=\mathrm{CO}(Z)$. Use countability of metrizable subsets of $Z$ (see [34]).

Corollary 4. There is a normal space which is collectionwise normal with respect to compact sets but is not collectionwise normal with respect to separable metric sets.

Proof. Let $Z$ be a Bernstein subset of the reals which is not Lebesgue measurable. Let $m$ be outer Lebesgue measure. Let $B=\mathrm{CO}(Z)$. Use the countability of compact sets and discrete families.

Corollary 5. Let $Z$ be a normal $\aleph_{1}$-compact non-Lindelöf (or normal countably compact noncompact) strongly zero-dimensional space (e.g., an ordinal of uncountable cofinality). There is a normal collectionwise Hausdorff space which is not collectionwise normal with respect to copies of $Z$.

Proof. Let $B$ be a clopen cover with no countable subcover. Let $m$ be the constant zero function.

Corollary 6. Let $Z$ be a normal countably compact strongly zero-dimensional space without isolated points. There is a normal collectionwise Hausdorff space which is not collectionwise normal with respect to copies of $Z$.

Proof. Any zero-dimensional space which has no isolated points can be mapped onto a dense subset of the Cantor set. Let $\psi: Z \rightarrow 2^{\omega}$ be such a continuous mapping with dense range. The mapping $\psi$ must be onto since $Z$ is countably compact. Let $B$ be the family of inverse images of open sets under this mapping. Let $m$ be defined such that $m\left(\psi^{-1}(U)\right)$ is the Lebesgue measure of $U$.

Next, we provide a lemma which sometimes removes the assumption of strong zero-dimensionality.

Lemma 15. Let $W$ be a normal collectionwise Hausdorff space and let $Z$ be a closed subspace of $W$. If there is a normal collectionwise Hausdorff space which is not collectionwise normal with respect to copies of $Z$, then there is a normal collectionwise Hausdorff space which is not collectionwise normal with respect to copies of $W$.

Proof. Let $X$ be a normal collectionwise Hausdorff space which is not collectionwise normal with respect to $\kappa$ many copies of $Z$. Without loss of generality, $X \supset Z \times \kappa$. Let $Y$ be the quotient space which is obtained by taking the free union of $W \times \kappa$ and $X$ and identifying each $(z, \alpha) \in X$ with $(z, \alpha) \in Z \times \kappa$. The quotient mapping is a perfect mapping and so $Y$ is normal. Of course, perfect mappings do not preserve collectionwise Hausdorff even 
in normal spaces (collapse the Cantor sets in Corollary 2), but $Y$ is collectionwise Hausdorff because the quotient mapping is finite-to-one. If the images of the $W \times\{\alpha\}$ were separated in $Y$, then the preimages of the open sets would separate the $Z \times\{\alpha\}$ in $X$, which is impossible.

Corollary 7. There is a normal collectionwise Hausdorff space which is not collectionwise normal with respect to copies of $[0,1]$.

Proof. Let $W=[0,1]$ and $Z=2^{\omega}$ and use Corollary 2 and Lemma 15.

The spaces of this section were constructed in 1982-83 while the author was visiting the University of Wisconsin at Madison. The author is thankful to Eric van Douwen for suggesting the use of strong zero-dimensionality to simplify the proof and for providing Corollary 6. The author thanks Bill Fleissner for finding an error in an earlier attempt at constructing this space. Fleissner has an alternate presentation of the spaces of this section. van Douwen has abstracted from the results of this section a machine which converts collectionwise Hausdorff spaces which are not collectionwise normal with respect to compact sets into normal collectionwise Hausdorff spaces which are not collectionwise normal with respect to compact sets.

We now return to Problem 7. We showed in [34] that normal collectionwise Hausdorff spaces are collectionwise normal with respect to paracompact scattered sets. This, together with Corollary 5 solves Problem 7 for ordinal spaces. If $\alpha$ is an ordinal, then collectionwise Hausdorff normal spaces are collectionwise normal with respect to copies of $\alpha$ if and only if $\alpha$ is a successor ordinal or a limit ordinal of countable cofinality.

Problem 8. Characterize those $A \subset \mathbb{R}$ for which there is a collectionwise Hausdorff normal space which is not collectionwise normal with respect to copies of A.

A set of reals $A$ has "strong measure zero" if, given any sequence $\left\{\varepsilon_{n}: n<\right.$ $\omega\}$ of positive reals, $A$ can be covered by a sequence of sets $X_{n}$ each having diameter less than $\varepsilon_{n}$.

Lemma 16. If $A \subset \mathbb{R}$ does not have strong measure zero, then there is a collectionwise Hausdorff normal space which is not collectionwise normal with respect to copies of $A$. If $A \subset \mathbb{R}$ is countable, then collectionwise Hausdorff normal spaces are collectionwise normal with respect to copies of $A$.

Any $A \subset \mathbb{R}$ which does not have strong measure zero has a strongly zerodimensional subset which also does not have strong measure zero. The proof of Example 7 works with any $Z \subset 2^{\omega}$ which does not have strong measure zero. Lemma 15 then implies that, if $A \subset \mathbb{R}$ has strong measure zero, then there is a collectionwise Hausdorff normal space which is not collectionwise normal with respect to copies of $A$. In [34], we showed that collectionwise Hausdorff normal spaces are collectionwise normal with respect to countable sets.

Corollary 8. It is consistent with the axioms of set theory that for each uncountable $A \subset \mathbb{R}$, there is a collectionwise Hausdorff normal space which is not collectionwise normal with respect to copies of $A$.

Proof. Laver showed in [18] that it is consistent that every strong measure zero is countable [18]. On the other hand Dow and the author have observed that 
if $A$ is a Lusin subset of the reals, then any normal collectionwise Hausdorff space is collectionwise normal with respect to copies of $A$. For more details see the author's upcoming paper Separation and point open games.

An even more general question may seem technical but would provide insight into the nature of certain categories of topological spaces.

Problem 9 [34]. Characterize those spaces $X$ and categories $\mathscr{C}$ for which there exists a normal space which is collectionwise normal with respect to discrete families of sets in $\mathscr{C}$ but not collectionwise normal with respect to copies of $X$.

Another question is a specific version of Problem 9:

Problem 10. Is there a normal not collectionwise normal space which is collectionwise normal with respect to collectionwise normal sets?

If the answer is no, then I would be amazed and it would solve Problems 19 and 18. If the answer is yes, then I think we would be getting at the heart of the topic of this section.

Problem 11. Is there a normal collectionwise Hausdorff space which is not collectionwise normal with respect to $\aleph_{1}$ many compact sets?

This question tries to get at the heart of the method of Example 7. Since measure is used in that argument, $Z$ has to be of positive measure. That means that $\kappa$ has to be big enough to list all the points of $Z$ and that means cardinality at least $2^{\omega}$. Thus an example answering Problem 11 would have to be essentially different from that of this paper and that would be very interesting. On the other hand, a theorem, under $\mathrm{MA}+\neg \mathrm{CH}$ for example, would be quite interesting.

Returning to Reed's results, the spaces of [23] are nonnormal but are first countable and even sometimes Moore spaces. He left open some questions, however:

Problem 12. Does ZFC imply that there is a collectionwise Hausdorff Moore space which is not collectionwise normal with respect to compact (metric) sets?

Reed only answered this affirmatively under the continuum hypothesis or Martin's axiom. The answer could well turn out to be yes since normality is not required and of course that would be preferable to Reed's results. If the answer is no, that is more interesting because the proof would penetrate into the manner in which the closed unit interval can be embedded in a Moore space and that would be quite exciting.

The spaces of this section have high character and leave us a long way from a solution to the next question.

Problem 13. Is it consistent that there is a normal first countable collectionwise Hausdorff space which is not collectionwise normal with respect to compact sets?

In fact, the two prototypes of normal collectionwise Hausdorff spaces which are not collectionwise normal have unseparated sets which are far from compact. Neither of these constructions is going to be easily modified to a positive solution to Problem 13. I do not think such a consistent example exists-it is asking 
too much. On the other hand, a negative answer means that rare thing: a ZFC result!

In fact, we are far from even partial results on the next general question:

Problem 14. Characterize those spaces $X$ for which there exists a consistent example of a normal collectionwise Hausdorff first countable space which is not collectionwise normal with respect to copies of $X$.

The only such $X$ which is known so far is the metric space $\omega_{1}^{\omega}$.

Problem 15. Does ZFC imply that normal first countable collectionwise Hausdorff spaces are collectionwise normal with respect to scattered sets?

This question asks whether Fleissner's space "George" can be modified to be first countable under some set-theoretic hypotheses. After all, Navy's space has been successfully modified to be first countable: that is Fleissner's solution to the normal Moore space conjecture $[13,10]$. This is a question which has great intrinsic interest. A consistent method which succeeds in lowering the character of the earlier prototype would undoubtedly be quite useful in many other contexts. A positive answer would be unthinkable. My interest in Problem 15 is heightened by the withdrawal in August 1989 of [14] in which a negative answer had been claimed (Fleissner circulated in late 1989 a manuscript in which a correct proof is claimed). That paper leads naturally to other related questions:

Problem 16. Does $V=L$ imply that normal first countable spaces are collectionwise normal with respect to separable sets?

Problem 17. Does $V=L$ imply that normal first countable spaces are collectionwise normal with respect to copies of $\omega_{1}$ ?

One is also led to Problems 16 and 17 in studying Fleissner's George [11], a normal collectionwise Hausdorff space which fails to be collectionwise normal with respect to copies of $\omega_{1}$. This space has been modified [32] to fail to be collectionwise normal with respect to separable sets ( $S$-spaces actually), under suitable set-theoretic assumptions. The example of [14] attempted to fail to be collectionwise normal with respect to copies of a space something like $\omega_{1}$. Problem 16 asks whether an $S$-space (like Ostaszewski's space [22]) can be used. Problem 17 asks whether it is really necessary to use something different from $\omega_{1}$ at all. Fleissner showed in 1977 [8] that it is consistent that normal first countable spaces are collectionwise normal with respect to copies of $\omega_{1}$ by collapsing an inaccessible in a model of the constructible universe (see also [6]). I think that the techniques that one would have to develop in order to solve these questions would be useful in many areas of general topology, and thus worth the effort.

\section{AdDitional PROPERTIES}

There are additional properties which the spaces of this paper may or may not have but which can be built in. The methods used are quite general and in this section theorems will be stated which enable us to get each of the following properties: 
2. locally collectionwise normal (or locally metrizable for Moore spaces)

3. perfect

without destroying many other properties.

Some of these theorems are statements of folklore: (1) is due to Michael and Bing and (3) is due to Bing. The approach in this section is preferable to building each of these properties into each of the spaces of this paper.

Lemma 17 (Splitting points). Let $X$ be a completely regular (or just $T_{1}$ ) space where $X=F \cup G, F$ is metacompact and $G$ is a set of isolated points. There is a metacompact space $Y=F \cup\left(G \times[F]^{2}\right)$ such that $F$ has the subspace topology, $Y-F$ is a set of isolated points and $X$ and $Y$ have identical separation properties.

Proof. For each open set $U$ in $X$, let $U^{*}=(U \cap F) \cup\{(g,\{x, y\}): g \in G \cap U$, $\{x, y\} \cap U \neq \varnothing\}$. Define a topology by letting each $U^{*}$ be open. Note that $(U \cap V)^{*} \subset U^{*} \cap V^{*}$ and that $(g,\{x, y\}) \in U^{*}$ implies $g \in U$ and so points are closed. To show that $X$ and $Y$ have identical separation properties, we suppose that $\left\{F_{\alpha}: \alpha \in \kappa\right\}$ is a discrete family of nonempty subsets of $F$. Suppose that $\left\{U_{\alpha}: \alpha \in \kappa\right\}$ is a disjoint family of open sets in $X$ such that each $U_{\alpha} \supset F_{\alpha}$. We claim that $\left\{U_{\alpha}^{*}: \alpha \in \kappa\right\}$ is a disjoint family of open sets in $Y$ such that each $U_{\alpha}^{*} \supset F_{\alpha}$. Suppose that $(g,\{x, y\}) \in U_{\alpha}^{*} \cap U_{\beta}^{*}$. We deduce that $g \in G \cap U_{\alpha} \cap U_{\beta}$ which is a contradiction.

Suppose that $\left\{\bigcup\left\{\left(U_{\alpha}^{\beta}\right)^{*}: \beta \in \lambda\right\}: \alpha \in \kappa\right\}$ is a disjoint family of open sets in $Y$ such that each $\bigcup\left\{\left(U_{\alpha}^{\beta}\right)^{*}: \beta \in \lambda\right\} \supset F_{\alpha}$. We can assume that each $U_{\alpha}^{\beta}$ intersects $F_{\alpha}$. We claim that $\left\{\bigcup\left\{U_{\alpha}^{\beta}: \beta \in \lambda\right\}: \alpha \in \kappa\right\}$ is a disjoint family of open sets in $X$ such that each $\bigcup\left\{U_{\alpha}^{\beta}: \beta \in \lambda\right\} \supset F_{\alpha}$. Suppose that $g \in U_{\alpha_{0}}^{\beta_{0}} \cap U_{\alpha_{1}}^{\beta_{1}} \cap G$. Choose $x_{i} \in U_{\alpha_{i}}^{\beta_{i}} \cap F_{\alpha}$. Now observe that $\left(g,\left\{x_{0}, x_{1}\right\}\right) \in$ $\left(U_{\alpha_{0}}^{\beta_{0}}\right)^{*} \cap\left(U_{\alpha_{1}}^{\beta_{1}}\right)^{*}$.

Suppose $\left\{U^{*}: U \in \mathscr{U}\right\}$ is an open cover of $Y$. Now $\{U \cap F: U \in \mathscr{U}\}$ is a relatively open cover of the subspace $F$. Since $F$ is metacompact, this relatively open cover has a point-finite open refinement $\mathscr{V}$. Since $F$ is closed, this refinement can be expressed as the restriction to $F$ of an open refinement $\mathscr{W}$ of $\mathscr{U}$. We claim that $\left\{W^{*}: W \in \mathscr{W}\right\}$ is a point-finite refinement of $\left\{U^{*}: U \in \mathscr{U}\right\}$. Suppose $(g,\{x, y\})$ is an element of each element of $\left\{\left(W_{n}\right)^{*}\right.$ : $n \in \omega\}$. This means that, for each $n \in \omega,\{x, y\} \cap W_{n} \cap F \neq \varnothing$. Without loss of generality, $x$ is an element of infinitely many $W_{n} \cap F$ which is a contradiction since these $W_{n} \cap F$ are elements of the point-finite family $\mathscr{V}$.

Note the use of this splitting points technique in Example 4.

Note that Lemma 17 can be modified. If $\left\{F_{\alpha}: \alpha \in \kappa\right\}$ is a discrete family of subsets of $F$, then $[F]^{2}$ can be replaced by $\left\{(x, y): x \in F_{\alpha}, y \in F_{\beta}, \alpha \neq \beta\right\}$. In this subspace $Z$ of $Y$, a family of subsets of a fixed $F_{\alpha}$ is separated in $Z$ if and only if it is separated in $F_{\alpha}$ and a family $\left\{A_{\alpha}: \alpha \in \kappa\right\}$ where $A_{\alpha} \subset F_{\alpha}$ is separated in $Z$ if and only if it is separated in $X \quad\left(U^{*} \cap V^{*} \neq \varnothing\right.$ implies that some $(g,\{x, y\}) \in U^{*} \cap V^{*}$ which implies $U \cap F_{\alpha} \neq \varnothing \wedge U \cap F_{\beta} \neq \varnothing$ for some $\alpha \neq \beta)$.

Corollary 9. If there is a nonmetrizable normal Moore space which is not collectionwise normal with respect to metrizable sets, then there is a metacompact nonmetrizable normal Moore space. 
Note that splitting points destroys local compactness and multiplies density by the cardinality of the underlying space but preserves character, pseudocharacter, cardinality, para-Lindelöf, Moore, perfect, etc.

Corollary 10. If there is a nonmetrizable normal Moore space which is not collectionwise normal with respect to metrizable sets, then there is a locally metrizable nonmetrizable normal Moore space.

Proof. Assume, without loss of generality, that $\left\{F_{\alpha}: \alpha \in \kappa\right\}$ partitions $F$. Then $\left\{F_{\alpha} \cup G: \alpha \in \kappa\right\}$ is an open cover of the subspace of $Y$ defined in the remarks following Lemma 17. Each $F_{\alpha} \cup G$ is a collectionwise normal (since $F_{\alpha}$ is collectionwise normal and even metrizable) Moore space and thus metrizable.

This technique appeared first implicitly in [19] and [3], then explicitly in [28] in the special case $X^{\prime \prime}=\varnothing$. The special case where $X$ is a Moore space appears in II.6 of [27].

Problem 18. Does the existence of a nonmetrizable normal Moore space imply the existence of a metacompact nonmetrizable normal Moore space?

Problem 19. Does the existence of a nonmetrizable normal Moore space imply the existence of a normal Moore space which is not collectionwise normal with respect to metrizable sets?

The technique of splitting points demonstrates that a positive answer to Problem 19 implies a positive answer to Problem 18. The reason these questions remain of interest is that a tremendous amount of effort has gone into obtaining partial positive results. Rudin and Starbird [24] and Nyikos [21] both obtained some technical results of great interest. Nyikos showed, in particular, that if there is a nonmetrizable normal Moore space, then there is a metacompact Moore space with a family of closed sets which is normalized but not separated. In [32], it is shown that, if there is a nonmetrizable normal Moore space which is nonmetrizable because it has a nonseparated discrete family of closed metrizable sets, then there is a metacompact nonmetrizable normal Moore space. A negative solution to either problem implies a positive solution to Problem 10 .

Lemma 18 (Perfection). Let $X$ be a completely regular space where $X=F \cup G$, $F$ is perfect and $G$ is a set of isolated points. There is a perfect space $Y=$ $F \cup(G \times \omega)$ such that $F$ has the subspace topology, $Y-F$ is a set of isolated points and $X$ and $Y$ have identical separation properties.

Proof. Let $U^{n}=(U \cap F) \cup\{(g, m): g \in U, m>n\}$ for each open set $U$ in $X$. Topologize $Y$ by letting each $U^{n}$ be open. Note that $U^{n} \cap V^{m}=\varnothing$ if and only if $U \cap V=\varnothing$. This immediately implies that $X$ and $Y$ have identical separation properties. We show that $Y$ is perfect. Let $A$ be a closed set, let $G_{i}$ be open sets in the subspace $F$ such that $\bigcap\left\{G_{i}: i \in \omega\right\}=A \cap F$. The set $\bigcap\left\{\left(G_{i} \cup G\right)^{i} \cup(A \cap G): i \in \omega\right\}$ intersects $F$ in $\bigcap\left\{G_{i}: i \in \omega\right\}=A \cap F$ and intersects $G$ in $\bigcap\left\{G_{i} \cup(A \cap G): i \in \omega\right\}=(A \cap G) \cup \cap\left\{G_{i}: i \in \omega\right\}=A \cap G$. Therefore, $\bigcap\left\{\left(G_{i} \cup G\right)^{i} \cup(A \cap G): i \in \omega\right\}=(A \cap F) \cup(A \cap G)=A$.

Note that perfecting destroys local compactness but preserves character, pseudocharacter, density, cardinality, metacompact, para-Lindelöf, Moore, etc. This technique first appeared in [2]. 


\section{REFERENCES}

1. Z. Balogh, Paracompactness in locally Lindelöf spaces, Canad. J. Math. 38 (1986), 719-727.

2. R. H. Bing, Metrization of topological spaces, Canad. J.'Math. 3 (1951), 175-186.

3. __ A translation of the normal Moore space conjecture, Proc. Amer. Math. Soc. 16 (1965), 612-619.

4. D. Burke, Covering properties, Handbook of Set-Theoretic Topology (Ken Kunen and Jerry Vaughan, eds.), North-Holland, Amsterdam, 1984, pp. 347-422.

5. F. S. Cater, P. Erdös, and F. Galvin, On the density of $\lambda$-box products, Gen. Topology Appl. 9 (1978), 307-312.

6. A. Dow, F. Tall, and W. Weiss, New proofs of the consistency of the normal Moore space conjecture. I, Topology Appl. 37 (1990), 33-51.

7. R. Engelking, General topology, PWN, Warsaw, 1977.

8. W. G. Fleissner, The character of $\omega_{1}$ in first countable spaces, Proc. Amer. Math. Soc. 62 (1977), 149-155.

9. __ A collectionwise Hausdorff, nonnormal space with a $\sigma$-locally countable base, Topology Proc. 4 (1979), 83-96.

10. __ If all normal Moore spaces are metrizable, then there is an inner model with a measurable cardinal, Trans. Amer. Math. Soc. 273 (1982), 365-373.

11. __ A normal, collectionwise Hausdorff, not collectionwise normal space, Gen. Topology Appl. 6 (1976), 57-71.

12. _ Normal Moore spaces in the constructible universe, Proc. Amer. Math. Soc. 46 (1974), 294-298.

13. _ _ Normal nonmetrizable Moore space from continuum hypothesis or nonexistence of inner models with measureable cardinals, Proc. Nat. Acad. Sci. U.S.A. 79 (1982), 13711372.

14. __ Son of George and $V=L$, J. Symbolic Logic 48 (1982), 71-77.

15. W. G. Fleissner and G. M. Reed, Para-Lindelöf spaces and spaces with a $\sigma$-locally countable base, Topology Proc. 2 (1977), 89-110.

16. G. Gruenhage, Paracompactness and subparacompactness in perfectly normal locally compact spaces, Russian Math. Surveys 35 (1980), 49-55.

17. T. Jech and K. Prikry, Confinality of the partial ordering of functions from $\omega_{1}$ into $\omega$ under eventual domination, Math. Proc. Cambridge Philos. Soc. 95 (1984), 25-32.

18. R. Laver, On the consistency of Borel's conjecture, Acta Math. 137 (1976), 151-169.

19. E. Michael, Point-finite and locally finite coverings, Canad. J. Math. 7 (1955), 275-279.

20. C. Navy, A paralindelöf space which is not paracompact, Ph.D. thesis, Univ. of WisconsinMadison, 1981.

21. P. J. Nyikos, Some normal Moore spaces, Colloq. Math. Soc. János Bolyai 23 (1978), 883ff.

22. A. J. Ostaszewski, On countably compact perfectly normal spaces, J. London Math. Soc. 14 (1976), 505-516.

23. G. M. Reed, Collectionwise Hausdorff versus collectionwise normal with respect to compact sets, Topology Appl. 16 (1983), 259-272.

24. M. E. Rudin and M. Starbird, Some examples of normal Moore spaces, Canad. J. Math. 29 (1977), 84-92.

25. J. Steprāns, Some results in set theory, Ph.D. thesis, Univ. of Toronto, 1982.

26. F. D. Tall, The density topology, Pacific J. Math. 62 (1976), 275-284.

27. _ Normality versus collectionwise normality, Handbook of Set-Theoretic Topology (K. Kunen and J. Vaughan, eds.), North-Holland, Amsterdam, 1984, pp. 685-733.

28. _ - On the existence of normal metacompact Moore spaces which are not metrizable, Canad. J. Math. 26 (1974), 1-6.

29. __ Set-theoretic consistency results and topological theorems concerning the normal Moore space conjecture and related problems, Dissertationes Math. 148 (1977), 1-53. 
30. P. Urysohn, Über die Mächtigkeit der zusammenhängenden Mengen, Math. Ann. 94 (1925), 262-295.

31. S. Watson, The character of Bing's space, Topology Appl. 28 (1988), 171-175.

32. __ Comments on separation, Topology Proc. 14 (1989), 315-372.

33. __ A construction of a Dowker space, Proc. Amer. Math. Soc. 109 (1990), 835-841.

34. __, Number versus size, Proc. Amer. Math. Soc. 102 (1988), 761-764.

35. N. H. Williams, Combinatorial set theory, North-Holland, Amsterdam, 1977.

Department of Mathematics, York University, North York, Ontario, Canada M3J 1PB 